\title{
MULTI-SIGHTLINE OBSERVATION OF NARROW ABSORPTION LINES IN LENSED QUASAR SDSS
} $\mathrm{J} 1029+2623^{*} \dagger$

\author{
Toru Misawa ${ }^{1}$, Cristian Saez ${ }^{2,3}$, Jane C. Charlton ${ }^{4}$, Michael Eracleous ${ }^{4,5}$, George Chartas ${ }^{6}$, Franz E. Bauer ${ }^{7,8,9}$, \\ NAOHISA INADA ${ }^{10}$, AND HisaKaZU UChIYAMA ${ }^{11}$ \\ ${ }^{1}$ School of General Education, Shinshu University, 3-1-1 Asahi, Matsumoto, Nagano 390-8621, Japan; misawatr@ shinshu-u.ac.jp \\ ${ }^{2}$ Korea Astronomy and Space Science Institute (KASI), 61-1, Hwaam-dong, Yuseong-gu, Deajeon 305-348, Korea \\ ${ }^{3}$ Department of Astronomy, University of Maryland, College Park, MD 20742-2421, USA \\ ${ }^{4}$ Department of Astronomy and Astrophysics, The Pennsylvania State University, University Park, PA 16802, USA \\ ${ }^{5}$ Center for Gravitational Wave Physics, The Pennsylvania State University, University Park, PA 16802, USA \\ ${ }^{6}$ Department of Physics and Astronomy, College of Charleston, SC 29424, USA
7 Instituto de Astrofísica, Facultad de Física, Pontificia Universidad Católica de Chile, Casilla 306, Santiago 22, Chile \\ ${ }^{8}$ Millennium Institute of Astrophysics (MAS), Nuncio Monseñor Sótero Sanz 100, Providencia, Santiago, Chile \\ ${ }^{9}$ Space Science Institute, 4750 Walnut Street, Suite 205, Boulder, Colorado 80301, USA \\ ${ }^{10}$ Department of Physics, National Institute of Technology, Nara College, Yamatokohriyama, Nara 639-1080, Japan \\ ${ }^{11}$ Department of Astronomy, School of Science, Graduate University for Advanced Studies, Mitaka, Tokyo 181-8588, Japan \\ Received 2016 February 25; revised 2016 April 16; accepted 2016 April 25; published 2016 June 27
}

\begin{abstract}
We exploit the widely separated images of the lensed quasar SDSS J1029+2623 $\left(z_{\mathrm{em}}=2.197, \theta=22\right.$ ". 5$)$ to observe its outflowing wind through two different sightlines. We present an analysis of three observations, including two with the Subaru telescope in 2010 February and 2014 April, separated by four years, and one with the Very Large Telescope, separated from the second Subaru observation by 2 months. We detect 66 narrow absorption lines (NALs), of which 24 are classified as intrinsic NALs that are physically associated with the quasar based on partial coverage analysis. The velocities of intrinsic NALs appear to cluster around values of $v_{\mathrm{ej}} \sim 59,000$, 43,000 , and $29,000 \mathrm{~km} \mathrm{~s}^{-1}$, which is reminiscent of filamentary structures obtained by numerical simulations. There are no common intrinsic NALs at the same redshift along the two sightlines, implying that the transverse size of the NAL absorbers should be smaller than the sightline distance between two lensed images. In addition to the NALs with large ejection velocities of $v_{\mathrm{ej}}>1000 \mathrm{~km} \mathrm{~s}^{-1}$, we also detect broader proximity absorption lines (PALs) at $z_{\mathrm{abs}} \sim z_{\mathrm{em}}$. The PALs are likely to arise in outflowing gas at a distance of $r \leqslant 620 \mathrm{pc}$ from the central black hole with an electron density of $n_{\mathrm{e}} \geqslant 8.7 \times 10^{3} \mathrm{~cm}^{-3}$. These limits are based on the assumption that the variability of the lines is due to recombination. We discuss the implications of these results on the three-dimensional structure of the outflow.
\end{abstract}

Key words: quasars: absorption lines - quasars: individual (SDSS J1029+2623)

Supporting material: figure set, machine-readable table

\section{INTRODUCTION}

Active galactic nucleus (AGN) outflows, potentially powered by one or more of a variety of mechanisms (e.g., radiation force, magnetic pressure, and magnetocentrifugal force), are important ingredients of quasar central engines and likely play a role in quasar and galaxy formation/evolution because: (1) they extract angular momentum from accretion disks, allowing gas accretion to proceed (e.g., Blandford \& Parne 1982; Emmering et al. 1992; Konigl \& Kartje 1994; Everett 2005), leading to the growth of black holes; (2) they also provide energy and momentum feedback to the interstellar medium of host galaxies and to the intergalactic medium (IGM), and inhibit star formation (e.g., Springel et al. 2005); and (3) they may promote metal enrichment of the IGM (e.g., Hamann et al. 1997a; Gabel et al. 2006). These outflowing gases, which are difficult to observe directly, have been detected as absorption lines in the spectra of about $50 \%$ of all quasars (e.g., Vestergaard 2003; Wise et al. 2004; Misawa et al. 2007a; Nestor et al. 2008; Muzahid et al. 2013). However, a limitation

\footnotetext{
* Based on data collected at Subaru Telescope, which is operated by the National Astronomical Observatory of Japan.

$\dagger$ Based on observations obtained at the European Southern Observatory at La Silla, Chile in programs 092.B-0512(A).
}

of these past studies is that they observe the outflowing gas only along a single sightline (i.e., one dimension) toward the nucleus of each quasar, although the absorber's physical conditions probably depend on the location/orientation at which we observe it (e.g., Elvis 2000; Ganguly et al. 2001). Thus, the internal structure of outflowing winds is still largely unknown.

Multiple quasar images, produced by gravitational lensing, provide a unique way to study the outflowing gas along more than one sightline. Lensed quasars with large image separation angles have a higher chance of revealing structural differences in the outflowing winds, especially in the vicinity of the continuum source. In this sense, the quasar images that are lensed by a cluster of galaxies (rather than a single massive galaxy) are very promising targets. Among three such lensed quasars, SDSS J1029+2623 at $z_{\mathrm{em}} \sim 2.197$ (Inada et al. 2006; Oguri et al. 2008) is the best target because (1) it has the largest lensed quasar image separation $(\theta \sim 22$ ". 5$)$ ever observed (see Figure 1 of Inada et al. 2006), and (2) it exhibits absorption features in the blue wings of the $\mathrm{C}$ Iv, $\mathrm{Nv}$, and $\mathrm{Ly} \alpha$ emission lines with ejection velocities of $v_{\mathrm{ej}} \leqslant 1000 \mathrm{~km} \mathrm{~s}^{-1}$, which could be the result of outflowing gas moving toward us from the central region. We call them proximity absorption lines (PALs) throughout this paper. We define PALs as a subcategory of 
narrow absorption lines (NALs) with ejection velocities of $v_{\mathrm{ej}} \leqslant$ $1000 \mathrm{~km} \mathrm{~s}^{-1}$. We use this terminology throughout the paper to separate PALs from NALs with larger ejection velocities.

Misawa et al. (2013) obtained high-resolution spectra of the brighter two of the lensed images (images A and B) with the Subaru telescope in 2010 February and found several clear signs that the origin of the PALs is indeed in the outflowing gas. First, they show the signature of partial coverage, which means the absorbers do not cover the background flux source completely. There also exists a clear difference in the absorption profiles between the spectra of images $\mathrm{A}$ and $\mathrm{B}$, which can be explained by either of the following two scenarios: (1) time variability of the absorption features over a timescale corresponding to the time delay between the two images (time variation scenario; Chartas et al. 2007) ${ }^{12}$; or (2) a difference in the absorption between the different sightlines of the outflowing wind (multi-sightline scenario; Chelouche 2003; Green 2006). However, with a single-epoch observation we cannot distinguish between these scenarios. Misawa et al. (2014b) performed a second observation about 4 years (1514 days in the observed frame) after the first observation (which is longer than the time delay between images $\mathrm{A}$ and $\mathrm{B} ; \Delta t_{\mathrm{AB}} \sim 744$ days), and found that the PALs were nearly stable and that most of the differences between images A and B still remained. This evidence suggests a multisightline scenario where the absorber's size should be smaller than the physical distance between the sightlines of the lensed images, thus not covering both sightlines. A possible explanation is that there are a number of small clumpy clouds in the outflowing stream. Indeed, some of the outflowing gas is expected to consist of small gas clouds $\left(d_{\text {cloud }} \leqslant 10^{-3} \mathrm{pc}\right)$ with very large gas densities $\left(n_{e} \geqslant 10^{6} \mathrm{~cm}^{-3}\right.$; Hamann et al. 2013; Joshi et al. 2014). Furthermore, recent radiationmagnetohydrodynamic (MHD) simulations by Takeuchi et al. (2013) reproduce variable clumpy structures with typical sizes of 20 times the gravitational radius $\left(R_{\mathrm{g}}\right)$, corresponding to $d_{\text {cloud }} \sim 5 \times 10^{-4} \mathrm{pc}$, assuming a black hole mass for $\operatorname{SDSS} \mathrm{J} 1029+2623$ of $M_{\mathrm{BH}} \sim 10^{8.72} \mathrm{M}_{\odot} \quad$ (Misawa et al. 2013).

Such clumpy clouds can be examined more easily through NALs with large offset velocities from the quasar because the corresponding absorbers are (1) probably smaller than the PAL absorbers, and (2) not so crowded in velocity space as the PAL absorbers. Indeed, there are many NALs detected in the spectra of both images A and B of SDSS J1029+2623. Their origin is not only the outflowing wind (intrinsic NALs hereafter) but also cosmologically intervening gas such as foreground galaxies and the IGM (intervening NALs hereafter). Although it has traditionally been believed that many NALs that fall within $5000 \mathrm{~km} \mathrm{~s}^{-1}$ of the quasar emission redshift (called associated absorption lines, or AALs) are physically associated with the quasar (e.g., Weymann et al. 1979), we can separate intrinsic NALs from intervening ones more effectively by performing partial coverage analysis. In order to form a global picture of the outflowing wind, we need to understand the physical conditions of NAL absorbers (i.e., highly accelerated gas) as well as PAL absorbers (i.e., weakly accelerated gas).

In this paper, we present the results from our new spectroscopic observation of SDSS J1029+2623 taken with

\footnotetext{
${ }^{12}$ The time delay between images $\mathrm{A}$ and $\mathrm{B}$ is $\Delta t_{\mathrm{AB}} \sim 774$ days in the sense of A leading $\mathrm{B}$, while the time delay between images $\mathrm{B}$ and $\mathrm{C} \Delta t_{\mathrm{BC}}$ is only a few days (Fohlmeister et al. 2013).
}

the Very Large Telescope (VLT), which enables us for the first time to identify intrinsic NALs in a high-quality spectrum of this object. In Section 2, we describe the observations and data reduction. The methods used for absorption line detection and covering factor analysis are outlined in Section 3. The results and discussion are presented in Section 4 and Section 5. Finally, we summarize our results in Section 6. We use a cosmology with $H_{0}=70 \mathrm{~km} \mathrm{~s}^{-1} \mathrm{Mpc}^{-1}, \quad \Omega_{m}=0.3$, and $\Omega_{\Lambda}=0.7$ throughout the paper.

\section{OBSERVATIONS}

We acquired high-resolution spectra of the brightest two of the three lensed images of SDSS J1029+2623, A and B with $V=18.72$ and 18.67 mags, with the VLT using the Ultraviolet and Visual Echelle Spectrograph (UVES) in queue mode (ESO program 092.B-0512(A)). The observations were performed from 2014 January 28 to February 26 (epoch E2 hereafter), which is $\sim 4$ years after the first observation on 2010 February 10 (epoch E1 hereafter; Misawa et al. 2013), and 2 months before the third observation on 2014 April 4 (epoch E3 hereafter; Misawa et al. 2014b) with Subaru using the High Dispersion Spectrograph (HDS). We used a slit width of 1"!2, corresponding to $R \sim 33,000$, while Misawa et al. (2013, 2014b) took $R \sim 30,000$ and 36,000 spectra using Subaru/ HDS. The wavelength coverage is $3300-6600 \AA$ in the $390 /$ $564 \mathrm{~nm}$ setting, which covers the $\mathrm{O}$ vi, $\mathrm{Nv}, \mathrm{Si}$ IV, and $\mathrm{C}$ IV doublets as well as the Ly $\alpha$ absorption line at $z_{\mathrm{abs}} \sim z_{\mathrm{em}}$. We also adopted $2 \times 2$ pixel binning in both the spatial and dispersion directions to increase the signal-to-noise ratio $(\mathrm{S} / \mathrm{N})$. The total integration time is $26,670 \mathrm{~s}$ and the final $\mathrm{S} / \mathrm{N}$ is about $23 \mathrm{pix}^{-1}$ around $4700 \AA$ for both images.

We reduced the data to extract the one-dimensional spectra in a standard manner using the UVES Common Pipeline Library (CPL release 6.6). We could not separate the third image (image $\mathrm{C}, V=20.63$ ) from image B completely because the typical seeing of our observation $(\sim 1$ !'0-1"! 8$)$ was comparable to the separation angle between images $\mathrm{B}$ and $\mathrm{C}$ $(\theta \sim 1 . " 85) .^{13}$

Table 1 gives a log of the current observation with VLT/ UVES as well as our past observations with Subaru/HDS, in which we list the target name, date of observation, telescope/ instrument used, spectral resolution, total exposure time, and $\mathrm{S} / \mathrm{N}$. The $\mathrm{S} / \mathrm{N}$ is evaluated around $4700 \AA$, close to the $\mathrm{C}$ IV mini-Broad absorption line (BAL). In Figure 1, we show normalized spectra over the full wavelength range of our observations for images A and B. These spectra were binned every $0.5 \AA$ for display purposes, and the $1 \sigma$ errors are also shown.

\section{DATA ANALYSIS}

First, using the line detection code SEARCH, written by Chris Churchill, we detect all absorption features whose confidence level is greater than $5 \sigma$ in the normalized spectrum of each lensed image. We then identify $\mathrm{N} v, \mathrm{C}$ IV, and $\mathrm{Si}$ IV doublets in the regions from $-1000 \mathrm{~km} \mathrm{~s}^{-1}$ to 5000 $\mathrm{km} \mathrm{s}^{-1}(\mathrm{~N} \mathrm{v})$, from $-1000 \mathrm{~km} \mathrm{~s}^{-1}$ to $70,000 \mathrm{~km} \mathrm{~s}^{-1}$ (C IV), and from $-1000 \mathrm{~km} \mathrm{~s}^{-1}$ to $40,000 \mathrm{~km} \mathrm{~s}^{-1}$ (Si IV) around the corresponding emission lines, with the maximum velocity set

\footnotetext{
13 On the other hand, a flux contamination from the image $\mathrm{C}$ is almost negligible in the image B spectrum because the former is much fainter than the latter.
} 
Table 1

Log of Observations

\begin{tabular}{|c|c|c|c|c|c|c|}
\hline Target & Obs Date & Instrument & $R$ & $\begin{array}{l}T_{\exp } \\
(\mathrm{s})\end{array}$ & $\begin{array}{c}\mathrm{S} / \mathrm{N}^{\mathrm{a}} \\
\left(\mathrm{pix}^{-1}\right)\end{array}$ & References $^{\mathrm{b}}$ \\
\hline \multirow[t]{2}{*}{ SDSS J1029+2623 A } & 2010 Feb 10 & Subaru/HDS & 30000 & 14400 & 13 & 1 \\
\hline & 2014 Apr 4 & Subaru/HDS & 36000 & 11400 & 14 & 3 \\
\hline \multirow[t]{2}{*}{ SDSS J1029+2623 B } & 2010 Feb 10 & Subaru/HDS & 30000 & 14200 & 13 & 1 \\
\hline & $2014 \mathrm{Feb} 4-26$ & VLT/UVES & 33000 & 26670 & 23 & 2 \\
\hline
\end{tabular}

Notes.

${ }^{a}$ Signal-to-noise ratio at $\lambda_{\text {obs }} \sim 4700 \AA$.

beferences. (1) Misawa et al. 2013, (2) This paper, (3) Misawa et al. 2014b.

in order to avoid the Ly $\alpha$ forest. ${ }^{14}$ We also search for the $\mathrm{Mg}$ II doublet in the whole range of the spectra. Absorption troughs that are separated by nonabsorbed regions are considered to be separate lines. In total, $4 \mathrm{Mg}$ II, $19 \mathrm{C} \mathrm{IV}, 2$ $\mathrm{Nv}$, and $7 \mathrm{Si}$ IV doublets are identified in the image $\mathrm{A}$ spectrum, while $3 \mathrm{Mg}$ II, $22 \mathrm{C}$ IV, $2 \mathrm{Nv}$, and $7 \mathrm{Si}$ IV doublets are identified in the image $\mathrm{B}$ spectrum. The equivalent widths of both blue and red members of doublets are measured for each line by integrating across the absorption profile, and these are listed in Table 2. We also searched for 10 single metal lines (O I $\lambda 1302$, Si II $\lambda 1190$, Si II $\lambda 1193$, Si II $\lambda 1260$, Si II $\lambda 1527, \quad A 1$ II $\lambda 1671, \quad C_{\text {II }} \lambda 1036, C_{\text {II }} \lambda 1335, \quad$ Si III $\lambda 1207$, and $\left.\mathrm{C}_{\mathrm{III}} \lambda 1548\right)$ as well as $\operatorname{Ly} \alpha$ and $\operatorname{Ly} \beta$ and detected about 200 lines at the same redshift as the doublet lines. These are summarized in Table 3. Other single lines or unidentified lines are not shown in Figure 1 and Tables $1-3$ even if they are detected at a confidence greater than $5 \sigma$.

\section{1. $d N / d z$ Analysis}

One of the important properties of intrinsic NALs is a number density excess of high-ionization doublets per unit redshift (i.e., $d N / d z$; Hamann et al. 1997b, and references therein). In order to compare the $d N / d z$ from our spectra with those from our previous study based on 37 quasar spectra (Misawa et al. 2007a), we construct a complete sample including only NALs whose blue doublet members would be detected even in the lowest $\mathrm{S} / \mathrm{N}$ spectrum in Misawa et al. (2007a). The corresponding lower limits of rest-frame equivalent widths (EWs) are $\mathrm{EW}_{\text {min }}^{\text {rest }}=0.056 \AA, 0.038 \AA$, and $0.054 \AA$ for $\mathrm{C}$ IV , N v, and Si IV, respectively. Here, the values of $\mathrm{EW}_{\text {min }}^{\text {rest }}$ depend on the $\mathrm{S} / \mathrm{N}$ of the observed spectrum as

$$
\begin{aligned}
& E W_{\min }^{\mathrm{rest}} \\
& =\frac{-U^{2}+U \sqrt{U^{2}+4(\mathrm{~S} / \mathrm{N})^{2}\left(M_{L}^{2} M_{c}^{-1}+M_{L}\right)}}{2(\mathrm{~S} / \mathrm{N})^{2}\left(1+z_{\mathrm{abs}}\right)} \\
& \quad \times \Delta \lambda(\AA),
\end{aligned}
$$

where $U$ is the confidence level of the EW defined as EW/ $\sigma(\mathrm{EW})$, and $M_{L}$ and $M_{C}$ are the numbers of pixels over which the EW and the continuum level are determined (Young et al. 1979; Tytler et al. 1987). Using Equation (1), we confirm that the $\mathrm{S} / \mathrm{N}$ of our VLT spectra is always larger than the

\footnotetext{
${ }^{14}$ Here, we define the velocity offset as positive for blueshifted NALs from the quasar emission redshift that is determined from the $\mathrm{Mg}$ II emission line (Inada et al. 2006).
}

required values except for the region between $\lambda_{\text {obs }} \sim$ 4500-4525 A (Figure 2). After removing weak NALs with $\mathrm{EW}^{\text {rest }}<\mathrm{EW}_{\text {min }}^{\text {rest }}$, we have $12 \mathrm{C}$ IV, $2 \mathrm{~N} \mathrm{v}$, and $3 \mathrm{Si}$ IV doublets in the image $\mathrm{A}$ spectrum and $11 \mathrm{CIV}, 2 \mathrm{Nv}$, and $3 \mathrm{Si}$ IV doublets in the image $\mathrm{B}$ spectrum. We will call this the "homogeneous" NAL sample hereafter. Following Misawa et al. (2007a), we also combined multiple NALs lying within $200 \mathrm{~km} \mathrm{~s}^{-1}$ of each other into a single NAL "system" because clustered lines are probably not independent even if they have a cosmologically intervening origin (e.g., Sargent et al. 1988).

All identified doublets (including both the homogeneous and inhomogeneous NAL samples) are listed in Table 2, in which multiple NALs within $200 \mathrm{~km} \mathrm{~s}^{-1}$ of each other are separated by horizontal lines. The table gives the ion name (ion), fluxweighted absorption redshift $\left(z_{\mathrm{abs}}\right)$, ejection velocity $\left(v_{\mathrm{ej}}\right)$ supposing they originate in the outflow, rest-frame EWs of blue and red members of the doublet $\left(\mathrm{EW}_{\mathrm{b}}^{\text {rest }}, \mathrm{EW}_{\mathrm{r}}^{\text {rest }}\right)$, identification number in Figure 1 (ID), reliability class of intrinsic lines (described later), ionization class (described later), and velocity difference from the first doublet at the lowest redshift in each absorption system $(\Delta v)$. Table 3 summarizes other information including the flux-weighted line width of each system on a velocity scale, $\sigma(v)$, as defined in Misawa et al. (2007a), and other transitions that are detected at the same redshift, as well as the column density $(\log N)$, Doppler parameter, $b$, and covering factor, $C_{\mathrm{f}}$, for each absorption component in the system.

\subsection{Partial Coverage Analysis}

Among several criteria, (1) time variability, (2) partial coverage, and (3) line-locking are the most reliable properties to distinguish intrinsic NALs from intervening NALs (Barlow \& Sargent 1997; Hamann et al. 1997b, and references therein). When compared with broad intrinsic absorption lines, intrinsic NALs are less likely to vary (Misawa et al. 2014a; Chen et al. 2015), and when they do vary, their variation amplitude is small (Wise et al. 2004; Misawa et al. 2014a). Therefore, the variability criterion does not offer an efficient way of identifying intrinsic NALs.

Partial coverage analysis is quite useful for our spectra because the resolving power is high enough to deblend NALs into multiple components. Using the Voigt profile fitting code MINFIT (Churchill 1997; Churchill et al. 2003), we deblended NALs into 86 and 91 components in images $\mathrm{A}$ and B, respectively. We do not include the $\mathrm{Si}$ IV NAL at $z_{\mathrm{abs}}=1.8909$ because the blue and red members of the doublet are both 

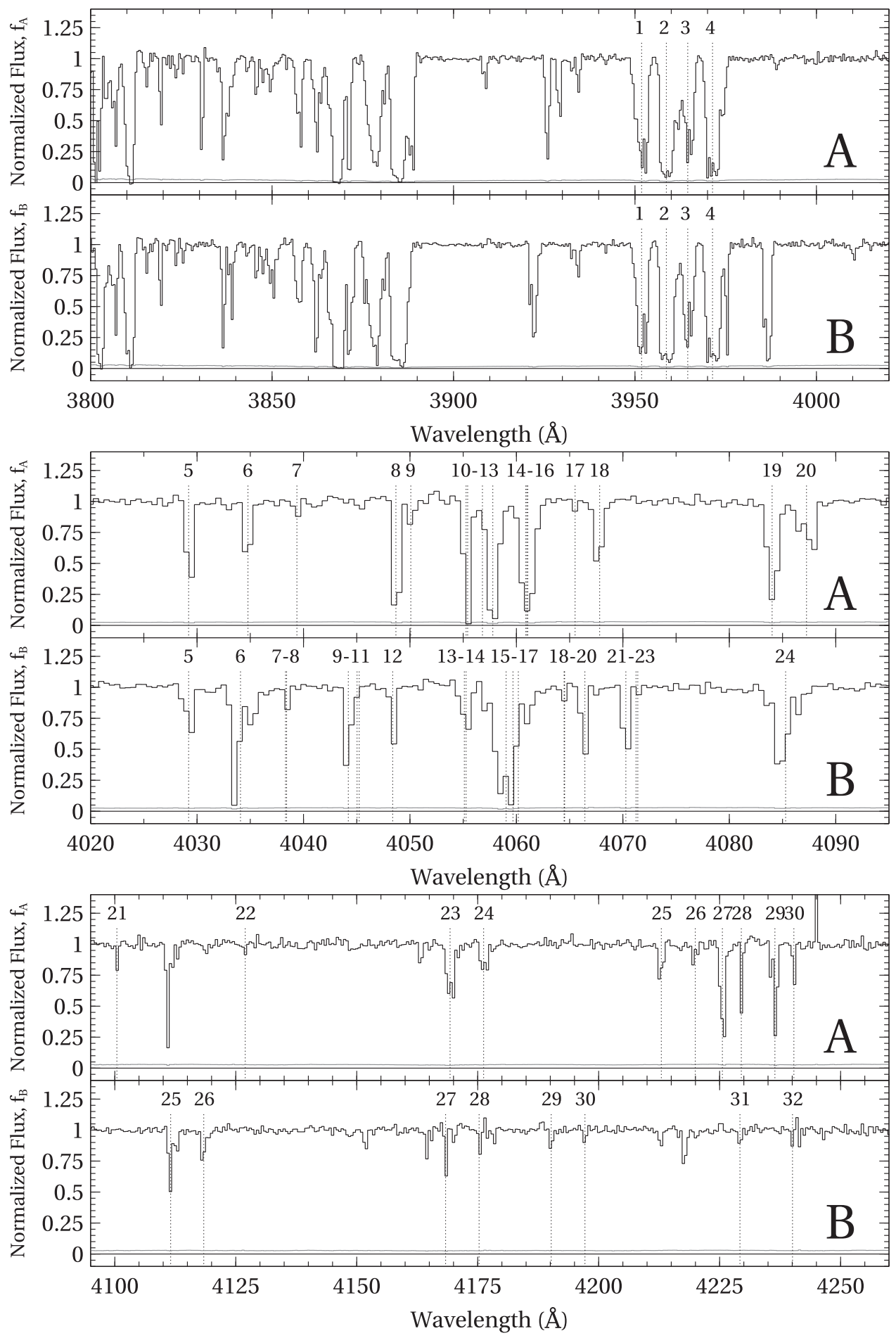

Figure 1. Normalized spectra and their $1 \sigma$ errors for images A and B of SDSS J1029+2623, taken with VLT/UVES, after sampling every $0.5 \AA$ for display purpose only.

blended with other lines. With MINFIT, we fit each NAL profile using the redshift (z), column density $\left(\log N\right.$ in $\left.\mathrm{cm}^{-2}\right)$, Doppler parameter $\left(b\right.$ in $\left.\mathrm{km} \mathrm{s}^{-1}\right)$, and covering factor $\left(C_{\mathrm{f}}\right)$ as free parameters. The covering factor $\left(C_{\mathrm{f}}\right)$ is the fraction of photons from the background source that pass through the absorber. If the background source is uniformly bright, then $C_{\mathrm{f}}$ also represents the fraction of the background source (i.e., the continuum source and/or broad emission line regions, BELRs) that is occulted by foreground absorbers along our sightline. If $C_{\mathrm{f}}$ is less than unity, it is likely that the absorbers are part of a quasar outflow because cosmologically intervening absorbers like substructures in foreground galaxies and the IGM are less likely to have internal structures as small as a size of the background flux sources (e.g., Wampler et al. 1995; Barlow \& Sargent 1997). The covering factor is evaluated in an unbiased manner as

$$
C_{f}=\frac{\left(R_{r}-1\right)^{2}}{1+R_{b}-2 R_{r}},
$$

where $R_{b}$ and $R_{r}$ are the residual (i.e., unabsorbed) fluxes of the blue and red members of a doublet in the normalized spectrum 


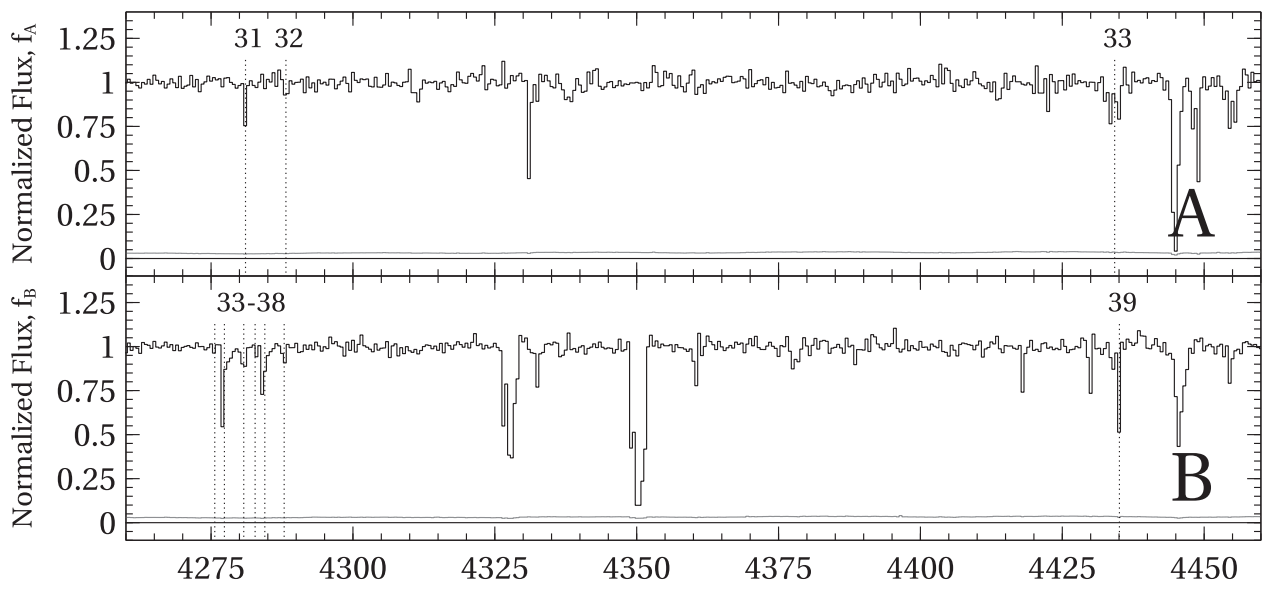

Wavelength $(\AA)$
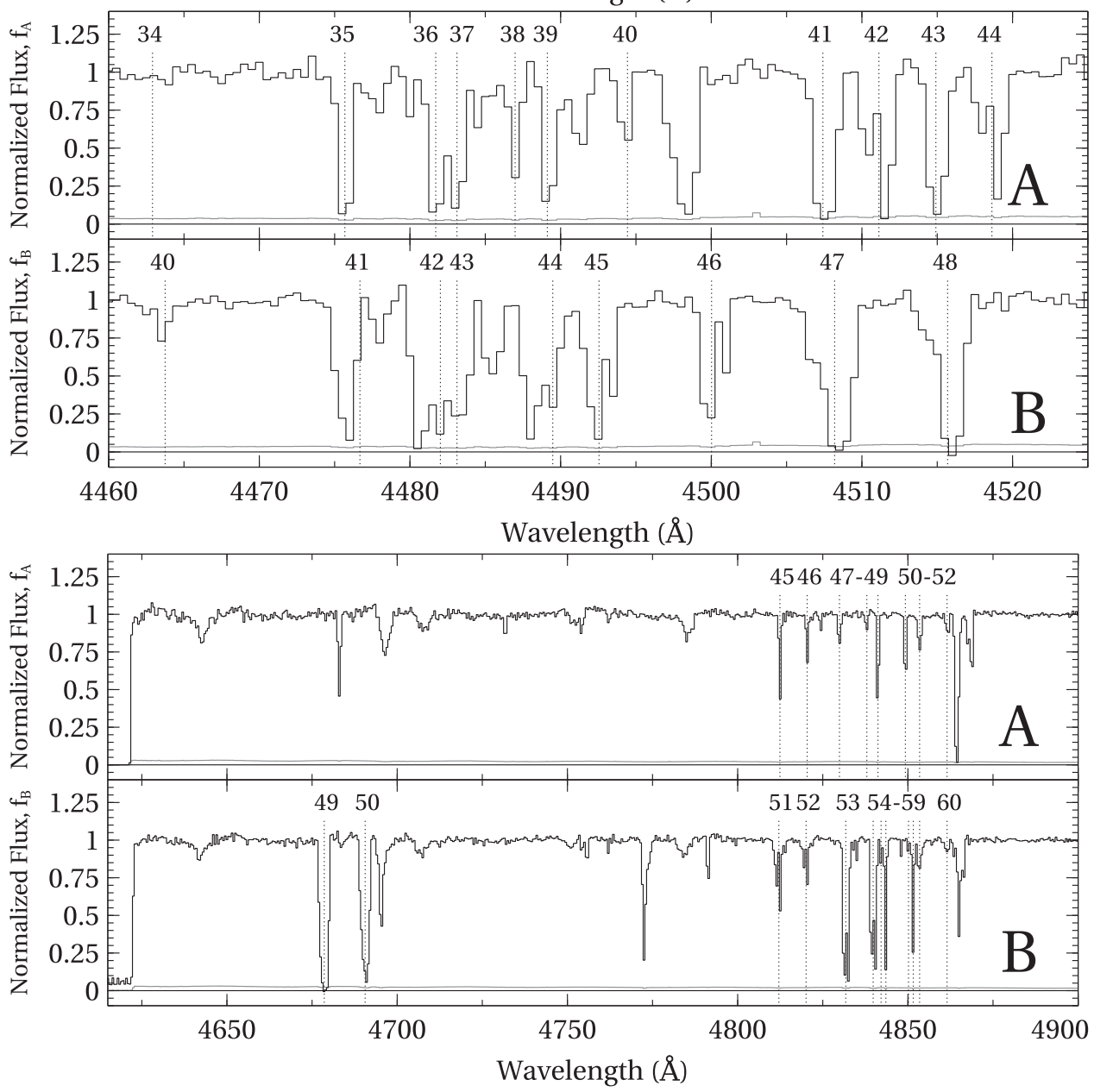

Figure 1. (Continued.)

(e.g., Barlow \& Sargent 1997; Hamann et al. 1997a; Crenshaw et al. 1999). If MINFIT gives unphysical covering factors for some components, e.g., negative or greater than 1 , we rerun the code assuming $C_{\mathrm{f}}=1$ only for those components because the $C_{\mathrm{f}}$ values are very sensitive to continuum level errors, especially for full coverage doublets (Misawa et al. 2005). In addition to the fitting method above, we evaluate $C_{\mathrm{f}}$ values for each pixel as Ganguly et al. (1999) did (pixel-by-pixel method). The fitting results by both methods are shown in Figure 3 and the fit parameters by the fitting method are summarized in Table 3 . We have confirmed that the $C_{\mathrm{f}}$ values provided by the two methods are in good agreement with each other.

\section{RESULTS}

\subsection{Narrow Absorption Lines}

Using our NAL sample toward the lensed images of SDSS $\mathrm{J} 1029+2623$, we perform several statistical analyses. In these 

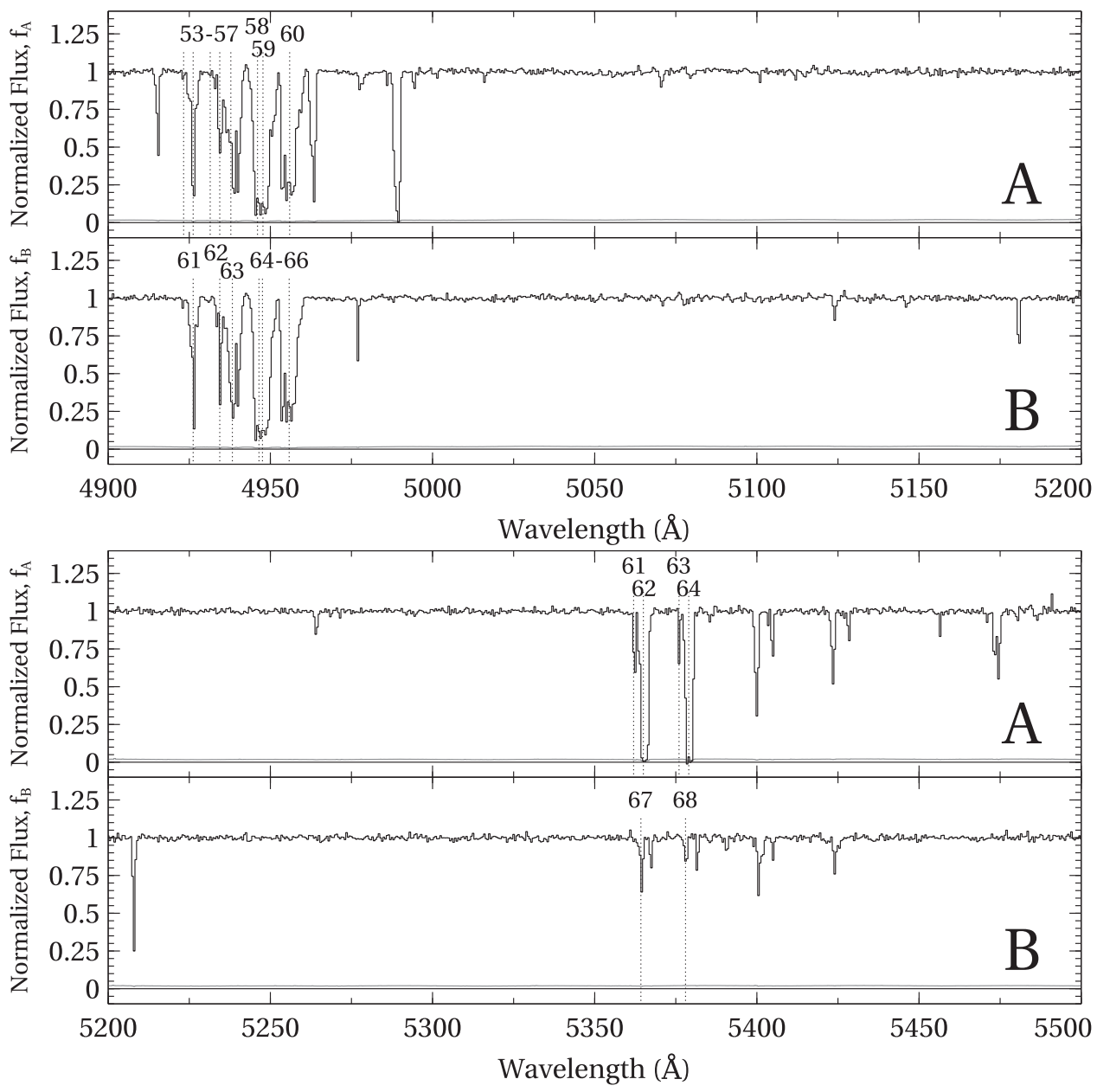

Figure 1. (Continued.)

analyses, in order to avoid any possible biases, we do not include the two C IV NALs at $z_{\mathrm{abs}} \sim 1.9322$ and 1.9788 that are covered in the Subaru spectrum (Misawa et al. 2013) but not covered by our VLT data.

\subsection{1. $d N / d z$ Analysis}

In Table 4, we summarize the number density of homogeneous NAL systems per unit redshift $(d N / d z)$ or per unit velocity offset from the quasar $(d N / d \beta)$ for $\mathrm{C}$ IV, $\mathrm{N}$ IV, and $\mathrm{Si}$ IV, along with the Poisson noise in these quantities (Gehrels 1986). All systems are also classified into one of two categories according to the offset velocity from the quasar; AALs with $v_{\mathrm{ej}}$ $\leqslant 5000 \mathrm{~km} \mathrm{~s}^{-1}$, and non-AALs with $v_{\mathrm{ej}}>5000 \mathrm{~km} \mathrm{~s}^{-1}$, following Misawa et al. (2007a). We found that the $d N / d z$ values for $\mathrm{C}$ IV, $\mathrm{N} \mathrm{v}$, and $\mathrm{Si}$ IV toward this one quasar, SDSS $\mathrm{J} 1029+2623$, are larger than the average values in the larger sample of Misawa et al. (2007a) by factors of $\sim 3, \sim 6$, and $\sim 2$, respectively, although this enhancement is not statistically significant because of the small number of NALs in our spectra. Because Misawa et al. (2007a) discovered that at least $20 \%$ of $\mathrm{C}$ IV NALs originate from winds based on partial coverage analysis, statistically we expect to detect two or more intrinsic $\mathrm{C}$ IV NALs among $\sim 10$ homogeneous $\mathrm{C}$ IV NALs in the spectra of images $\mathrm{A}$ and $\mathrm{B}$.

\subsubsection{Intrinsic or Intervening NALs}

High-velocity NALs blueshifted with $v_{\mathrm{ej}}>1000 \mathrm{~km} \mathrm{~s}^{-1}$ are expected to be outside of the range of velocities where BELs are found, and thus they absorb mostly continuum light. The existence of partial coverage in high-velocity NALs suggests that the size of absorbers is comparable to or smaller than the continuum source (i.e., $d_{\text {cloud }} \leqslant R_{\text {cont }}$ ). Following Misawa et al. (2007a), we separate all NALs (including those in the inhomogeneous sample) into three classes (classes A, B, and C) based on partial coverage analysis, where class A includes NALs most reliably classified as intrinsic while class C includes NALs that are consistent with full coverage or that cannot be classified. Class B contains NALs that show linelocking, which is a signature of a radiatively driven outflowing wind and is only detectable if our sightline is approximately parallel to the gas motion, as often seen in NALs (e.g., Benn et al. 2005; Bowler et al. 2014). Class B also contains systems that have tentative evidence for partial coverage. As a result, 4 $\mathrm{C}$ IV NALs (including PALs) are classified as intrinsic (2 class $\mathrm{A}$ and 2 class B) among the $12 \mathrm{C}$ IV NALs in the homogeneous sample from the spectrum of image $\mathrm{A}$, while $3 \mathrm{C}$ IV NALs are classified as intrinsic ( 2 class A and 1 class B) among 11 NALs in the spectrum of image B. The fraction of intrinsic NALs $(27 \%-33 \%)$ is somewhat larger than the average value of $\sim 20 \%$ (Misawa et al. 2007a), although our sample size is small. If we include the inhomogeneous sample, $5 \mathrm{C}$ IV NALs are 
Table 2

Absorption Systems with Doublet Lines

\begin{tabular}{|c|c|c|c|c|c|c|c|c|c|c|c|c|c|c|c|c|}
\hline \multicolumn{8}{|c|}{ Image A } & \multicolumn{8}{|c|}{ Image B } & \multirow[b]{2}{*}{$\begin{array}{c}\Delta v^{\mathrm{e}} \\
\left(\mathrm{km} \mathrm{s}^{-1}\right)\end{array}$} \\
\hline Ion & $z_{\mathrm{abs}}$ & $\begin{array}{c}v_{\mathrm{ej}} \\
\left(\mathrm{km} \mathrm{s}^{-1}\right)\end{array}$ & $\begin{array}{c}\mathrm{EW}_{\mathrm{b}}^{\text {resta }} \\
(\AA)\end{array}$ & $\begin{array}{c}\mathrm{EW}_{\mathrm{r}}^{\text {restb }} \\
(\AA)\end{array}$ & ID & Class- $1^{c}$ & Class- $2^{\mathrm{d}}$ & Ion & $z_{\mathrm{abs}}$ & $\begin{array}{c}v_{\mathrm{ej}} \\
\left(\mathrm{km} \mathrm{s}^{-1}\right)\end{array}$ & $\begin{array}{c}\mathrm{EW}_{\mathrm{b}}^{\text {resta }} \\
(\AA)\end{array}$ & $\begin{array}{c}\mathrm{EW}_{\mathrm{r}}^{\text {restb }} \\
(\AA \AA)\end{array}$ & ID & Class $-1^{c}$ & ${\text { Class }-2^{\mathrm{d}}}^{\mathrm{d}}$ & \\
\hline \multirow[t]{2}{*}{$\mathrm{Mg}$ II } & 0.5111 & 190431 & $0.604 \pm 0.008$ & $0.495 \pm 0.008$ & 27,29 & $\mathrm{C} 1$ & $\mathrm{~L}$ & & & & & & & & & \\
\hline & 0.5125 & 190265 & $0.221 \pm 0.005$ & $0.145 \pm 0.004$ & 28,30 & $\mathrm{C} 1$ & $\mathrm{~L}$ & $\mathrm{Mg}$ II & 0.5124 & 190276 & $0.095 \pm 0.004$ & $0.052 \pm 0.004$ & 31,32 & $\mathrm{C} 3$ & $\mathrm{~L}$ & $\begin{array}{r}0.0 \\
+19.8\end{array}$ \\
\hline $\mathrm{Mg}$ II & & & & & & & & $\mathrm{Mg}$ II & 0.6731 & 171003 & $1.689 \pm 0.008$ & $1.444 \pm 0.008$ & 49,50 & $\mathrm{C} 1$ & $\mathrm{~L}$ & \\
\hline $\mathrm{Mg}$ II & 0.9187 & $\begin{array}{l}141245 \\
141116\end{array}$ & $\begin{array}{l}0.193 \pm 0.002 \\
1.472 \pm 0.006\end{array}$ & $\begin{array}{l}0.120 \pm 0.002 \\
1.366 \pm 0.006\end{array}$ & $\begin{array}{l}61,63 \\
62,64\end{array}$ & $\begin{array}{l}\mathrm{C} 3 \\
\mathrm{C} 1\end{array}$ & $\mathrm{~L}$ & $\mathrm{Mg}$ II & 0.9184 & 141157 & $0.186 \pm 0.005$ & $0.092 \pm 0.005$ & 67,68 & $\mathrm{C} 1$ & $\mathrm{~L}$ & $\begin{array}{r}0.0 \\
+125.1 \\
+172.0\end{array}$ \\
\hline $\mathrm{Mg}$ II & & & & & & & & C Iv & 1.6085 & 60206 & $0.035 \pm 0.002$ & $0.026 \pm 0.002$ & 7,10 & B2 & $\mathrm{H}$ & \\
\hline $\begin{array}{l}\text { C IV } \\
\text { C IV }\end{array}$ & $\begin{array}{l}1.6151 \\
1.6160\end{array}$ & $\begin{array}{l}59472 \\
59378\end{array}$ & $\begin{array}{l}0.308 \pm 0.003 \\
0.047 \pm 0.003\end{array}$ & $\begin{array}{c}0.297 \pm 0.003 \\
\quad \leqslant 0.033\end{array}$ & $\begin{array}{l}8,11 \\
9,12\end{array}$ & $\begin{array}{c}\mathrm{B} 2, \mathrm{H} \\
\mathrm{C} 2\end{array}$ & $\mathrm{IH}$ & C Iv & 1.6149 & 59493 & $0.105 \pm 0.004$ & $\leqslant 0.132$ & 12,13 & B2 & $\mathrm{IH}$ & $\begin{array}{r}0.0 \\
+22.9 \\
+126.2\end{array}$ \\
\hline \multirow[t]{3}{*}{$\mathrm{C}$ IV } & 1.6230 & 58601 & $\leqslant 0.512$ & $0.190 \pm 0.007$ & 16,18 & $\mathrm{~B} 2, \mathrm{H}$ & $\mathrm{H}$ & C IV & 1.6221 & 58699 & $\leqslant 0.284$ & $0.144 \pm 0.002$ & 16,20 & $\mathrm{C} 2, \mathrm{H}$ & $\mathrm{H}$ & $\begin{array}{r}0.0 \\
+103.0\end{array}$ \\
\hline & & & & & & & & C Iv & 1.6253 & 58350 & $0.021 \pm 0.002$ & $0.014 \pm 0.002$ & 18,22 & B2 & $\mathrm{H}$ & \\
\hline & & & & & & & & $\mathrm{C}$ IV & 1.6556 & 55033 & $0.133 \pm 0.004$ & $0.081 \pm 0.003$ & 25,26 & $\mathrm{C} 1$ & $\mathrm{H}$ & \\
\hline \multirow[t]{2}{*}{$\mathrm{C}_{\mathrm{IV}}$} & 1.6930 & 50965 & $0.498 \pm 0.005$ & $0.185 \pm 0.007$ & 23,24 & $\mathrm{C} 2, \mathrm{H}$ & LIH & C Iv & 1.6924 & 51029 & $0.084 \pm 0.002$ & $0.046 \pm 0.002$ & 27,28 & $\mathrm{C} 2$ & $\mathrm{H}$ & $\begin{array}{r}0.0 \\
+66.8\end{array}$ \\
\hline & & & & & & & & C Iv & 1.7065 & 49509 & $0.042 \pm 0.002$ & $0.032 \pm 0.002$ & 29,30 & $\mathrm{~A} 2$ & $\mathrm{H}$ & \\
\hline \multirow[t]{2}{*}{$\mathrm{C}_{\mathrm{IV}}$} & 1.7212 & 47932 & $0.493 \pm 0.005$ & $0.053 \pm 0.004$ & 25,26 & $\mathrm{C} 2, \mathrm{H}$ & $\mathrm{H}$ & & & & & & & & & \\
\hline & & & & & & & & $\begin{array}{l}\mathrm{C}_{\text {IV }} \\
\mathrm{C}_{\text {IV }}\end{array}$ & $\begin{array}{l}1.7617 \\
1.7627\end{array}$ & $\begin{array}{l}43596 \\
43496\end{array}$ & $\begin{array}{l}0.010 \pm 0.002 \\
0.094 \pm 0.002\end{array}$ & $\begin{array}{l}0.011 \pm 0.002 \\
0.066 \pm 0.002\end{array}$ & $\begin{array}{l}33,36 \\
34,37\end{array}$ & $\begin{array}{l}\text { C3 } \\
\text { B1 }\end{array}$ & LIH & $\begin{array}{r}0.0 \\
+108.6\end{array}$ \\
\hline C IV & 1.7652 & 43224 & $0.047 \pm 0.002$ & $0.035 \pm 0.002$ & 31,32 & A2 & $\mathrm{LIH}$ & C IV & 1.7650 & 43250 & $0.041 \pm 0.002$ & $0.022 \pm 0.002$ & 35,38 & $\mathrm{~A} 2$ & $\mathrm{H}$ & $\begin{array}{r}0.0 \\
+21.7\end{array}$ \\
\hline C IV & 1.8909 & 30091 & $0.355 \pm 0.004$ & $0.309 \pm 0.003$ & 35,37 & $\mathrm{C} 1, \mathrm{H}$ & LIH & & & & & & & & & 0.0 \\
\hline \multirow[t]{2}{*}{ Si IV } & 1.8909 & 30088 & $0.181 \pm 0.003$ & $\leqslant 0.268$ & 5,10 & B2, H & & C IV & 1.8909 & 30088 & $0.456 \pm 0.006$ & $\leqslant 0.501$ & 41,43 & $\mathrm{C} 2, \mathrm{H}$ & $\mathrm{IH}$ & $\begin{array}{l}0.0 \\
0.0\end{array}$ \\
\hline & & & & & & & & Si IV & 1.8909 & 30087 & $0.121 \pm 0.004$ & $0.119 \pm 0.004$ & 5,14 & B2 & & 0.0 \\
\hline $\begin{array}{l}\text { C IV } \\
\text { Si IV }\end{array}$ & $\begin{array}{l}1.8949 \\
1.8949\end{array}$ & $\begin{array}{l}29686 \\
29685\end{array}$ & $\begin{array}{l}0.413 \pm 0.004 \\
0.137 \pm 0.003\end{array}$ & $\begin{array}{c}0.318 \pm 0.004 \\
\quad \leqslant 0.464\end{array}$ & $\begin{array}{c}36,39 \\
6,14\end{array}$ & $\begin{array}{c}\mathrm{C} 2, \mathrm{H} \\
\mathrm{B} 2\end{array}$ & LIH & $\begin{array}{l}\mathrm{Si} \text { IV } \\
\mathrm{C} \text { IV }\end{array}$ & $\begin{array}{l}1.8944 \\
1.8945\end{array}$ & $\begin{array}{l}29737 \\
29723\end{array}$ & $\begin{array}{c}0.380 \pm 0.005 \\
\quad \leqslant 0.669\end{array}$ & $\begin{array}{l}\leqslant 0.383 \\
\leqslant 0.592\end{array}$ & $\begin{array}{l}6,17 \\
42,44\end{array}$ & $\begin{array}{l}\mathrm{C} 2, \mathrm{H} \\
\mathrm{C} 2, \mathrm{H}\end{array}$ & LIH & $\begin{array}{r}0.0 \\
+10.4 \\
+51.8 \\
+51.8\end{array}$ \\
\hline $\begin{array}{l}\mathrm{C} \text { IV } \\
\mathrm{Si} \text { IV }\end{array}$ & $\begin{array}{l}1.8982 \\
1.8982\end{array}$ & $\begin{array}{l}29343 \\
29342\end{array}$ & $\begin{array}{l}0.193 \pm 0.004 \\
0.029 \pm 0.003\end{array}$ & $\begin{array}{l}0.146 \pm 0.005 \\
0.013 \pm 0.002\end{array}$ & $\begin{array}{c}38,40 \\
7,17\end{array}$ & $\begin{array}{c}\mathrm{C} 1, \mathrm{H} \\
\mathrm{C} 2\end{array}$ & $\mathrm{IH}$ & Si IV & 1.8975 & 29413 & $0.032 \pm 0.002$ & $0.019 \pm 0.002$ & 8,19 & B2 & LIH & $\begin{array}{r}0.0 \\
+72.5 \\
+72.5\end{array}$ \\
\hline Si IV & & & & & & & & Si IV & 1.9016 & 28991 & $0.162 \pm 0.003$ & $0.142 \pm 0.002$ & 9,21 & $\mathrm{C} 1, \mathrm{H}$ & LIH & 0.0 \\
\hline
\end{tabular}


Table 2

(Continued)

\begin{tabular}{|c|c|c|c|c|c|c|c|c|c|c|c|c|c|c|c|c|}
\hline \multicolumn{8}{|c|}{ Image A } & \multicolumn{8}{|c|}{ Image B } & \multirow[b]{2}{*}{$\begin{array}{c}\Delta v^{e} \\
\left(\mathrm{~km} \mathrm{~s}^{-1}\right)\end{array}$} \\
\hline Ion & $z_{\mathrm{abs}}$ & $\begin{array}{c}v_{\mathrm{ej}} \\
\left(\mathrm{km} \mathrm{s}^{-1}\right)\end{array}$ & $\begin{array}{c}\mathrm{EW}_{\mathrm{b}}^{\text {resta }} \\
(\AA)\end{array}$ & $\begin{array}{c}\mathrm{EW}_{\mathrm{r}}^{\text {restb }} \\
(\AA)\end{array}$ & ID & Class- $1^{\mathrm{c}}$ & Class- $2^{\mathrm{d}}$ & Ion & $z_{\text {abs }}$ & $\begin{array}{c}v_{\mathrm{ej}} \\
\left(\mathrm{km} \mathrm{s}^{-1}\right)\end{array}$ & $\begin{array}{c}\mathrm{EW}_{\mathrm{b}}^{\text {resta }} \\
(\AA)\end{array}$ & $\begin{array}{c}\mathrm{EW}_{\mathrm{r}}^{\text {restb }} \\
(\AA)\end{array}$ & ID & Class- $1^{\mathrm{c}}$ & Class- $2^{\mathrm{d}}$ & \\
\hline & & & & & & & & C IV & 1.9019 & 28964 & $0.457 \pm 0.005$ & $0.363 \pm 0.006$ & 45,46 & $\mathrm{C} 1, \mathrm{H}$ & & +31.0 \\
\hline & & & & & & & & Si IV & 1.9024 & 28917 & $0.023 \pm 0.002$ & $0.013 \pm 0.002$ & 11,23 & B2 & & +82.7 \\
\hline $\mathrm{C}_{\text {IV }}$ & 1.9115 & 27981 & $0.572 \pm 0.008$ & $0.448 \pm 0.007$ & 41,43 & $\mathrm{C} 1, \mathrm{H}$ & LIH & & & & & & & & & 0.0 \\
\hline \multirow[t]{3}{*}{ Si IV } & 1.9115 & 27987 & $0.439 \pm 0.004$ & $0.318 \pm 0.004$ & 13,19 & $\mathrm{C} 1, \mathrm{H}$ & & & & & & & & & & 0.0 \\
\hline & & & & & & & & $\mathrm{Si}$ IV & 1.9118 & 27949 & $\leqslant 0.248$ & $\leqslant 0.197$ & 15,24 & $\mathrm{C} 2, \mathrm{H}$ & & +30.9 \\
\hline & & & & & & & & C IV & 1.9119 & 27946 & $0.837 \pm 0.009$ & $0.679 \pm 0.009$ & 47,48 & $\mathrm{C} 1, \mathrm{H}$ & LIH & +41.2 \\
\hline $\mathrm{C}_{\mathrm{IV}}$ & 1.9138 & 27745 & $0.484 \pm 0.009$ & $0.359 \pm 0.009$ & 42,44 & $\mathrm{C} 3, \mathrm{H}$ & & & & & & & & & & +236.9 \\
\hline Si IV & 1.9141 & 27718 & $\leqslant 0.461$ & $0.205 \pm 0.005$ & 15,20 & $\mathrm{~B} 2, \mathrm{H}$ & LIH & & & & & & & & & +267.8 \\
\hline \multirow[t]{2}{*}{ Si IV } & 1.9420 & 24880 & $0.041 \pm 0.002$ & $0.019 \pm 0.003$ & 21,22 & $\mathrm{C} 2$ & LI & & & & & & & & & \\
\hline & 2.1084 & 8426 & $0.130 \pm 0.002$ & $0.074 \pm 0.002$ & 45,46 & $\mathrm{C} 2$ & $\mathrm{IH}$ & $\mathrm{C}_{\text {IV }}$ & 2.1081 & 8460 & $0.209 \pm 0.004$ & $0.110 \pm 0.004$ & 51,52 & $\mathrm{~B} 1, \mathrm{H}$ & $\mathrm{IH}$ & $\begin{array}{r}0.0 \\
+29.0\end{array}$ \\
\hline \multirow[t]{2}{*}{$\mathrm{C}_{\text {IV }}$} & 2.1196 & 7348 & $\leqslant 0.065$ & $0.024 \pm 0.001$ & 47,48 & $\mathrm{C} 2$ & LH & & & & & & & & & 0.0 \\
\hline & & & & & & & & C IV & 2.1209 & 7223 & $0.552 \pm 0.003$ & $0.470 \pm 0.003$ & 53,54 & $\mathrm{C} 1, \mathrm{H}$ & LIH & +125.0 \\
\hline \multirow[t]{3}{*}{ C Iv } & 2.1270 & 6643 & $0.145 \pm 0.001$ & $0.114 \pm 0.001$ & 49,50 & $\mathrm{C} 1$ & $\mathrm{H}$ & & & & & & & & & 0.0 \\
\hline & & & & & & & & $\mathrm{C}_{\text {IV }}$ & 2.1276 & 6585 & $0.029 \pm 0.002$ & $0.014 \pm 0.002$ & 55,57 & $\mathrm{C} 2$ & $\mathrm{IH}$ & +57.5 \\
\hline & & & & & & & & C Iv & 2.1285 & 6500 & $0.183 \pm 0.002$ & $0.155 \pm 0.002$ & 56,58 & $\mathrm{C} 1, \mathrm{H}$ & & +143.9 \\
\hline \multirow[t]{2}{*}{$\mathrm{C}_{\mathrm{IV}}$} & 2.1349 & 5884 & $0.093 \pm 0.003$ & $0.046 \pm 0.002$ & 51,52 & $\mathrm{C} 1$ & $\mathrm{H}$ & & & & & & & & & 0.0 \\
\hline & & & & & & & & C IV & 2.1349 & 5886 & $0.073 \pm 0.002$ & $0.032 \pm 0.002$ & 59,60 & $\mathrm{C} 2$ & $\mathrm{H}$ & 0.0 \\
\hline C rv & 2.1800 & 1604 & $0.011 \pm 0.001$ & $0.008 \pm 0.001$ & 53,55 & $\mathrm{C} 2$ & $\mathrm{IH}$ & & & & & & & & & 0.0 \\
\hline Si IV & 2.1815 & 1458 & $0.115 \pm 0.006$ & $0.041 \pm 0.005$ & 33,34 & $\mathrm{C} 1$ & & & & & & & & & & +141.5 \\
\hline \multirow{3}{*}{$\mathrm{C}_{\text {IV }}$} & & & & & & & & $\mathrm{C}_{\text {Iv }}$ & 2.1818 & 1428 & $0.298 \pm 0.002$ & $0.209 \pm 0.002$ & 61,62 & $\mathrm{C} 1, \mathrm{H}$ & LIH & +169.7 \\
\hline & 2.1819 & 1416 & $0.425 \pm 0.004$ & $\leqslant 0.297$ & 54,56 & $\mathrm{C} 1, \mathrm{H}$ & & & & & & & & & & +179.2 \\
\hline & & & & & & & & Si Iv & 2.1821 & 1403 & $0.088 \pm 0.002$ & $0.062 \pm 0.003$ & 39,40 & $\mathrm{C} 1$ & & +198.0 \\
\hline & & & & & & & & C IV & 2.1897 & 686 & $0.848 \pm 0.003$ & $\leqslant 1.405$ & 63,64 & $\mathrm{~A} 1, \mathrm{H}$ & $\mathrm{H}$ & 0.0 \\
\hline C IV & 2.1898 & 676 & $0.902 \pm 0.003$ & $\leqslant 1.253$ & 57,58 & $\mathrm{~A} 1, \mathrm{H}$ & $\mathrm{H}$ & & & & & & & & & +9.4 \\
\hline \multirow[t]{2}{*}{$\mathrm{N} v$} & 2.1900 & 657 & $0.945 \pm 0.004$ & $\leqslant 0.882$ & 1,3 & $\mathrm{~A} 1, \mathrm{H}$ & & & & & & & & & & +28.2 \\
\hline & & & & & & & & $\mathrm{N} v$ & 2.1900 & 658 & $0.888 \pm 0.004$ & $\leqslant 0.717$ & 1,3 & $\mathrm{~A} 1, \mathrm{H}$ & & +28.2 \\
\hline \multirow[t]{3}{*}{$\mathrm{N} \mathrm{v}$} & 2.1955 & 141 & $\leqslant 1.494$ & $1.341 \pm 0.004$ & 2,4 & $\mathrm{~A} 1, \mathrm{H}$ & $\mathrm{H}$ & & & & & & & & & 0.0 \\
\hline & & & & & & & & $\mathrm{N} \mathrm{v}$ & 2.1955 & 141 & $\leqslant 1.231$ & $1.201 \pm 0.003$ & 2,4 & $\mathrm{~A} 1, \mathrm{H}$ & $\mathrm{H}$ & 0.0 \\
\hline & & & & & & & & $\mathrm{C}_{\mathrm{IV}}$ & 2.1957 & 122 & $1.569 \pm 0.003$ & $1.224 \pm 0.003$ & 65,66 & $\mathrm{~A} 1, \mathrm{H}$ & & +18.8 \\
\hline $\mathrm{C}_{\text {IV }}$ & 2.1958 & 113 & $\leqslant 1.590$ & $1.303 \pm 0.003$ & 59,60 & $\mathrm{~A} 1, \mathrm{H}$ & & & & & & & & & & +28.2 \\
\hline
\end{tabular}

Notes.

${ }^{\text {a }}$ Rest-frame equivalent width of the blue component of doublet line.

${ }^{\mathrm{b}}$ Rest-frame equivalent width of the red component of doublet line.

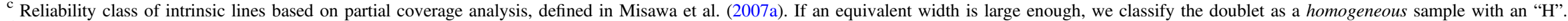

d Ionization class of absorption system with high (H; IP $>60 \mathrm{eV}$ ), intermediate (I; IP $=35-50 \mathrm{eV}$ ), and/or low (L; IP $<25 \mathrm{eV}$ ) ionization transitions.

${ }^{\mathrm{e}}$ Velocity difference from the first doublet in each absorption system. 
classified as 3 class A and 2 class B among 19 NALs toward image A, while 9 out of $22 \mathrm{C}$ IV NALs are classified into 4 class $A$ and 5 class B NALs toward image B. In addition to intrinsic C IV NALs, we detected two class B Si IV NALs only toward image A in homogeneous sample, and three class B Si IV NALs in each of the image A and B spectra after including systems from the inhomogeneous sample. It is also noteworthy that we detect a large number of line-locked NALs: $2 \mathrm{C}$ IV and $3 \mathrm{Si}$ IV NALs in image $\mathrm{A}$ and $5 \mathrm{C}$ IV and $3 \mathrm{Si}$ IV NALs in image $\mathrm{B}$, while only 5 systems are line-locked among 138 homogeneous NALs toward 37 quasars (Misawa et al. 2007a). This result strongly suggests that our sightline toward the central source is almost parallel to the outflowing streamline.

\subsubsection{Ionization Conditions}

The outflowing winds in the vicinity of the flux source are probably more highly ionized than most intervening absorbers due to strong UV radiation from the continuum source, although it depends on the gas density of the absorbers. Indeed, a high-ionization state has been used as one indicator of the intrinsic properties of absorbers (Hamann et al. 1997b, and references therein). Broad absorption lines (BALs) are often classified into three categories according to their ionization level: high-ionization BALs (HiBALs), low-ionization BALs (LoBALs), and extremely low-ionization BALs showing Fe II lines (FeLoBALs; Weymann et al. 1991). A similar classification has also been performed for NALs (Bergeron et al. 1994; Misawa et al. 2007a). Motivated by the literature, we classify our NALs into three categories based on the detection of absorption lines in low- (ionization potential; IP $<25 \mathrm{eV}$ ), intermediate- $(\mathrm{IP}=35-50 \mathrm{eV})$, and high-ionization (IP > $60 \mathrm{eV}$ ) levels. $^{15}$ Interestingly, some class A NAL systems include low-ionization lines (see Table 2), which suggests that intrinsic NAL absorbers have multiple phases with different ionization states, as noted in Misawa et al. (2007a).

\subsubsection{Similarities in NALs Between Sightlines}

Here, we present a new method of identifying intrinsic NALs by exploiting our multi-sightline observation. If NALs with similar line profiles are detected at the same redshift toward two sightlines, the corresponding absorber must have a size larger than the physical distance between the two sightlines. The absorber also cannot have any internal structures on the scale of the sightline separation. Although foreground galaxies and IGM structures also can cover both sightlines (whose physical separation is approximately kiloparsec or megaparsec scale), they usually have significant internal velocity structures on those scales, as often seen in the spectra of lensed quasars (e.g., Ellison et al. 2004). Only intrinsic absorbers can satisfy the requirement of having very similar profiles because their sizes can be larger than the sightline separation (e.g., sub-parsec scale) if they are at a small distance from the flux source (e.g., $r<1 \mathrm{kpc})$. Based on the ejection velocity distribution of classes A, B, and C NALs (Figure 4), in Figure 5 we summarize the distribution of velocity differences between NALs and PALs in the two sightlines $(\Delta v)$ for systems within $\Delta v \leqslant 200 \mathrm{~km} \mathrm{~s}^{-1}$ between the two sightlines. The distribution

\footnotetext{
15 An important caveat here is that we do not necessarily detect all absorption lines because of the detection limits due to line strength, observed wavelength coverage, and line blending (e.g., in Ly $\alpha$ forest).
}

is almost uniform up to $\Delta v \sim 200 \mathrm{~km} \mathrm{~s}^{-1}$ with a clear peak near $\Delta v \sim 0 \mathrm{~km} \mathrm{~s}^{-1}$. Among eight NAL pairs with $\Delta v \leqslant 10 \mathrm{~km}$ $\mathrm{s}^{-1}$, four are $\mathrm{C}$ IV and $\mathrm{N} v$ PALs at $z_{\mathrm{abs}} \sim z_{\mathrm{em}}$, which we will discuss later. The other four NAL pairs are C IV NALs at $z_{\mathrm{abs}} \sim$ $1.8909,2.1349$, and 2.1819, and a Si IV pair at $z_{\text {abs }} \sim 1.8909$. Although the velocity shift is very small for these NAL pairs, their line profiles are clearly different as compared in Figure 6. This means our two sightlines go through different absorbers or different regions of a single absorber. In either event, we cannot conclude that these are intrinsic NALs based only on this analysis. On the other hand, it is intriguing that no NAL pairs that are classified into classes $\mathrm{A} / \mathrm{B}$ have a common ejection velocity or line profile. For example, the $\mathrm{C}_{\text {IV }}$ NALs at $z_{\text {abs }}=1.7652$ toward image $\mathrm{A}$ and at $z_{\mathrm{abs}}=1.7650$ toward image B are both classified as class A NALs. Although their ejection velocities are very close each other, their line centers and profiles are obviously different, as shown in Figure 7. We also note that the difference is remarkable in all three epochs (see Figure 8). This means the multi-sightline scenario (i.e., that two sightlines pass through different regions of the outflow) is also applicable for intrinsic NALs with large ejection velocity as well as for PALs as already confirmed in Misawa et al. (2013, 2014b).

\subsubsection{Time Variability of NALs}

Because of the lower data quality $\left(\mathrm{S} / \mathrm{N} \sim 10\right.$ pixel $\left.^{-1}\right)$ and narrower effective wavelength coverage of the Subaru/HDS spectra taken in epochs E1 and E3, we cannot monitor NALs for time variability analysis with only a few exceptions. As an example, we compare spectra obtained at three epochs around the strong C IV NALs at $z_{\mathrm{abs}}=1.8909-1.9138(\mathrm{ID}=35-44)$ in image $A$ and $C$ IV NALs at $z_{\mathrm{abs}}=1.8909-1.9119$ (ID = 41-48) in image B as shown in Figure 9. These NALs are obviously not variable, which is consistent with the past result that NALs with large ejection velocities are rarely variable (Chen et al. 2015).

\subsection{Proximity Absorption Lines}

The absorption line group at $z_{\mathrm{abs}} \sim z_{\mathrm{em}}$ with an ejection velocity of $v_{\mathrm{ej}}<1000 \mathrm{~km} \mathrm{~s}^{-1}$ has already been observed twice in 2010 February (epoch E1) and 2014 April (epoch E3) with Subaru/HDS. Its origin is probably in the outflowing gas because (1) there is evidence for partial coverage (e.g., there exists a clear residual flux at the bottom of the $\operatorname{Ly} \alpha$ and $\mathrm{N} \mathrm{v}$ absorption lines even though they appear to be saturated); (2) the profiles are variable; and (3) the profiles show signatures of line-locking (Misawa et al. 2013, 2014b). The most important result from these past observations is that the absorption profiles of the Ly $\alpha, \mathrm{Nv}$, and $\mathrm{C}$ IV PALs in the spectra of images A and B are clearly different, especially for lines at $v_{\mathrm{ej}}<0 \mathrm{~km} \mathrm{~s}^{-1}$ (see Figure 10). There are at least three possible origins for the difference: (1) a micro-lensing effect, (2) time delay between the images, and (3) different absorber structure along the different sightlines. Among these, the first idea is immediately rejected because the lensed images show a common ratios between the radio, optical, and X-ray fluxes (Oguri et al. 2012; Ota et al. 2012), which is not expected for micro-lensing. We can also reject the time delay effect because the variability is almost negligible between epochs E1 and E3 (Misawa et al. 2014b). A difference in column density between the two sightlines is the only acceptable 
Table 3

Line Parameters of Narrow Absorption Components

\begin{tabular}{|c|c|c|c|c|c|c|c|}
\hline Ion & $\begin{array}{c}\lambda_{\text {obs }}{ }^{\mathrm{a}} \\
(\AA)\end{array}$ & $z_{\mathrm{abs}}{ }^{\mathrm{b}}$ & $\begin{array}{c}v_{\mathrm{ej}}^{\mathrm{c}} \\
\left(\mathrm{km} \mathrm{s}^{-1}\right)\end{array}$ & $\begin{array}{c}\log N \\
\left(\mathrm{~cm}^{-2}\right)\end{array}$ & $\begin{array}{c}\sigma(v) / b^{\mathrm{d}} \\
\left(\mathrm{km} \mathrm{s}^{-1}\right)\end{array}$ & $C_{\mathrm{f}}^{\mathrm{e}}$ & $\begin{array}{l}\text { Other } \\
\text { Ions }^{f}\end{array}$ \\
\hline \multicolumn{8}{|c|}{ Image A } \\
\hline \multirow[t]{5}{*}{$\mathrm{Mg}$ II } & 4225.6 & 0.5111 & 190431 & & 47.3 & & $\operatorname{Mg}$ I $\lambda 2853, \mathrm{Fe}$ пा $\lambda 2600$ (Fe ІІ $\lambda 2383)$ \\
\hline & & 0.5108 & 190464 & $12.96 \pm 0.06$ & $5.1 \pm 0.2$ & $0.93_{-0.06}^{+0.07}$ & \\
\hline & & 0.5112 & 190426 & $13.51 \pm 0.05$ & $10.5 \pm 0.5$ & $1.00_{-0.06}^{+0.06}$ & \\
\hline & & 0.5112 & 190423 & $13.69 \pm 0.33$ & $37.1 \pm 7.2$ & $0.19_{-0.09}^{+0.10}$ & \\
\hline & & 0.5113 & 190408 & $12.60 \pm 0.02$ & $7.6 \pm 0.3$ & 1.00 & \\
\hline \multirow[t]{3}{*}{$\mathrm{Mg}$ II } & 4229.5 & 0.5125 & 190265 & & 17.9 & & $\operatorname{Mg}$ І $\lambda 2853, \mathrm{Fe}$ ІІ $\lambda 2600$ (Fe ІІ $\lambda 2344, \mathrm{Fe}$ ІІ $\lambda 2383)$ \\
\hline & & 0.5125 & 190269 & $12.69 \pm 0.02$ & $6.0 \pm 0.4$ & 1.00 & \\
\hline & & 0.5126 & 190261 & $12.70 \pm 0.27$ & $13.3 \pm 4.2$ & $0.83_{-0.28}^{+0.46}$ & \\
\hline \multirow[t]{2}{*}{$\mathrm{Mg}$ II } & 5376.1 & 0.9176 & 141245 & & 14.1 & & $\operatorname{Mg}$ I $\lambda 2853, \mathrm{Fe}$ ІІ $\lambda 2600$ (A1 III $\lambda 1863)$ \\
\hline & & 0.9176 & 141246 & $12.95 \pm 0.02$ & $4.7 \pm 0.2$ & 1.00 & \\
\hline \multirow[t]{4}{*}{$\mathrm{Mg}$ II } & 5365.3 & 0.9187 & 141116 & & 68.1 & & $\operatorname{Mg}_{\text {I }} \lambda 2853, \mathrm{Fe}$ ІІ $\lambda 2344,2600(\mathrm{Al}$ ІІІ $\lambda 1863)$ \\
\hline & & 0.9181 & 141190 & $12.50 \pm 0.02$ & $4.1 \pm 0.3$ & 1.00 & \\
\hline & & 0.9185 & 141146 & $14.03 \pm 0.02$ & $16.9 \pm 0.2$ & 1.00 & \\
\hline & & 0.9189 & 141094 & $14.94 \pm 0.07$ & $22.0 \pm 0.5$ & $0.99_{-0.03}^{+0.03}$ & \\
\hline
\end{tabular}

Notes.

${ }^{\text {a }}$ Wavelength of flux-weighted line center.

${ }^{\mathrm{b}}$ Redshift of flux-weighted line center.

${ }^{\mathrm{c}}$ Ejection velocity from quasar emission redshift.

${ }^{\mathrm{d}}$ Flux-weighted line width $(\sigma(v))$ or Doppler parameter $(b)$.

e Covering factor.

${ }^{\mathrm{f}}$ Other single lines that are detected in the system. Lines in parentheses are in Ly $\alpha$ forest.

${ }^{\mathrm{g}}$ Two $\mathrm{C}_{\text {Iv PALs at }} z_{\mathrm{abs}} \sim 2.1898$ and 2.1958 are fitted together as a single absorption profile whose flux-weighted redshift is $z_{\text {abs }} \sim 2.1937$.

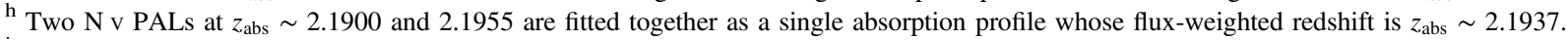

${ }^{i}$ We cannot fit this Si IV NAL because both members of the doublet are blending with other lines.

${ }^{\mathrm{j}}$ Two C Iv PALs at $z_{\mathrm{abs}} \sim 2.1897$ and 2.1957 are fitted together as a single absorption profile whose flux-weighted redshift is $z_{\mathrm{abs}} \sim 2.1937$.

${ }^{\mathrm{k}}$ Two N v PALs at $z_{\mathrm{abs}} \sim 2.1900$ and 2.1955 are fitted together as a single absorption profile whose flux-weighted redshift is $z_{\mathrm{abs}} \sim 2.1935$.

(This table is available in its entirety in machine-readable form.)

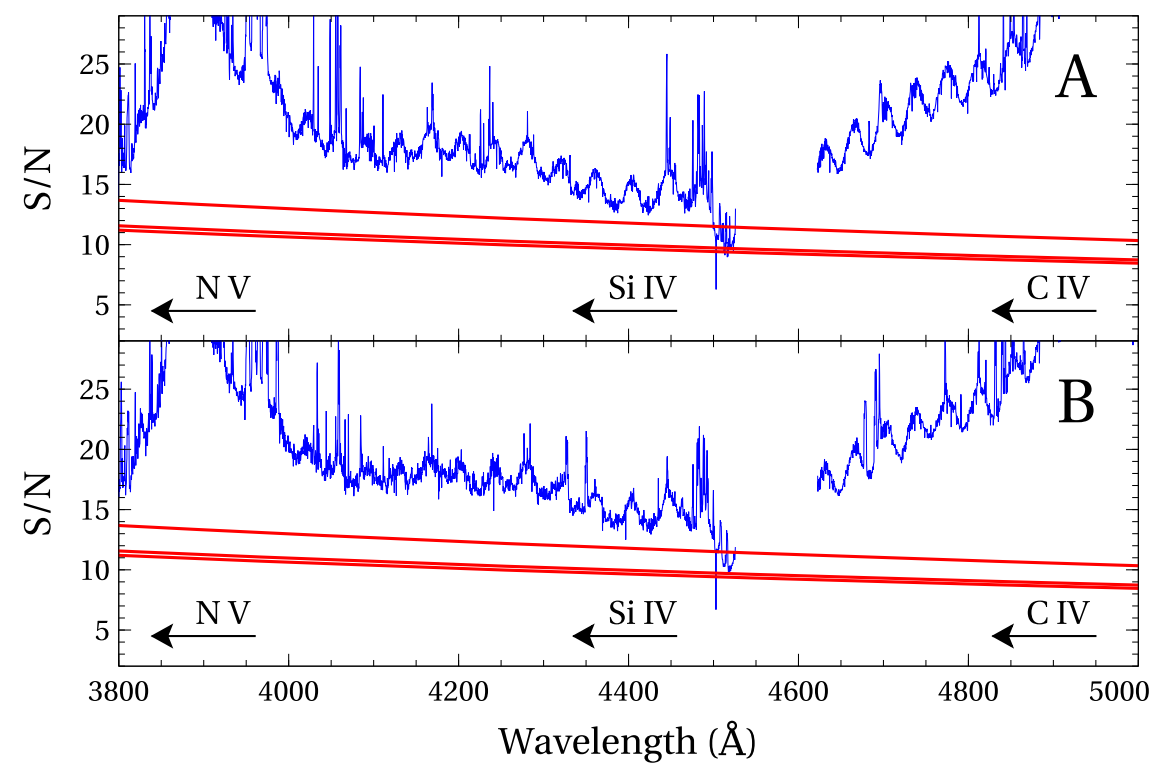

Figure 2. $\mathrm{S} / \mathrm{N}$ of the image $\mathrm{A}$ and $\mathrm{B}$ spectra (blue histograms) and the minimum $\mathrm{S} / \mathrm{N}$ ratio that is necessary for detecting homogeneous sample lines for $\mathrm{N} v$, $\mathrm{C}$ iv, and $\mathrm{Si}$ IV, respectively (red lines from top to bottom; see Section 3.1).

explanation. To our knowledge, this is the first time that an outflowing wind has been observed along multiple sightlines. Hereafter, we call the PALs at $v_{\mathrm{ej}}<0 \mathrm{~km} \mathrm{~s}^{-1}$ (showing sightline difference) narrow PALs and those at $v_{\mathrm{ej}}>0 \mathrm{~km} \mathrm{~s}^{-1}$ (showing similar line profiles between sightlines) broad PALs (see Figure 10).

Our observation with VLT/UVES not only gives an additional epoch for monitoring the PALs but enables us to study the 


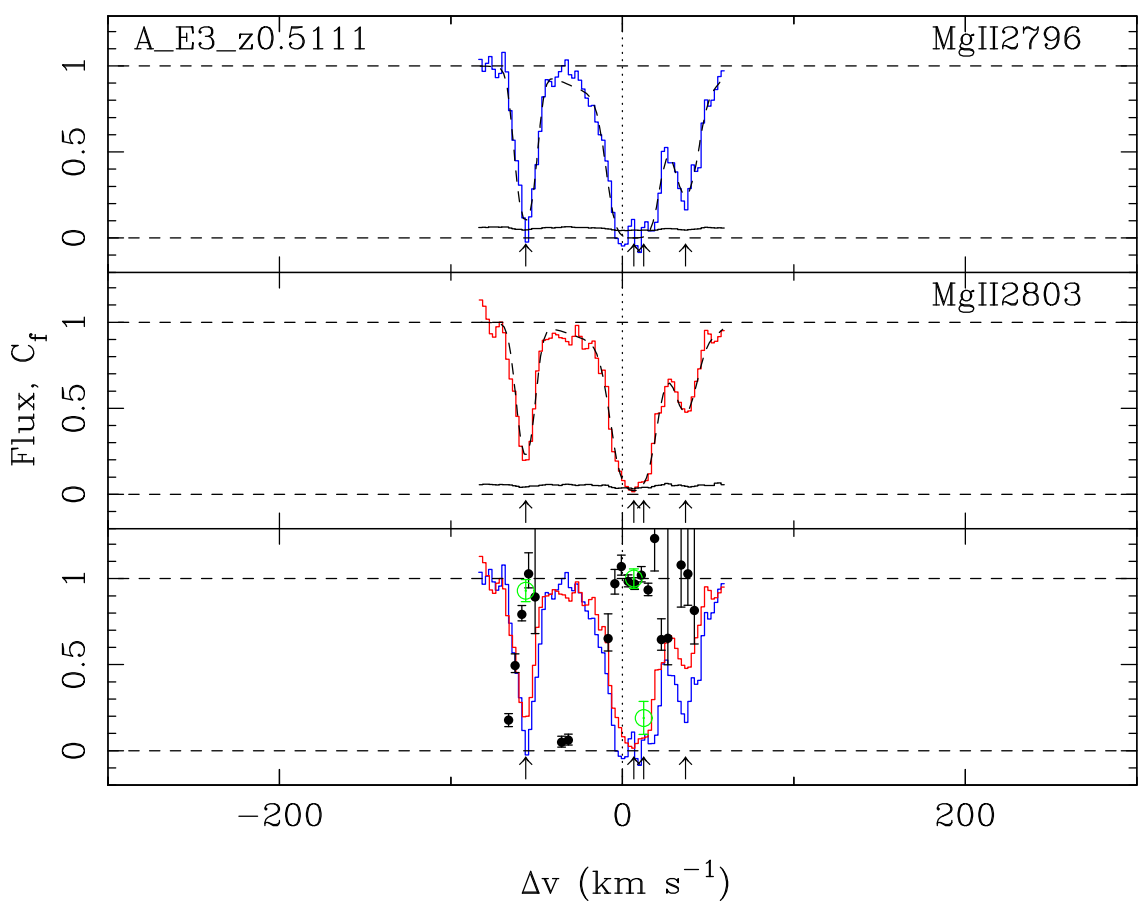

Figure 3. Results of partial coverage analysis applied to all C Iv, N v, Si Iv, and Mg II NALs detected in images A and B of SDSS J1029+2623, except for the PALs whose results are presented in Figure 12. The horizontal axis denotes the relative velocity from the flux-weighted center of the system $(\Delta v)$ while the vertical axis is the normalized flux. The first two panels show the profiles of the blue and red members of a doublet (blue and red histograms) with the model profile produced by MINFIT superimposed (dashed line). The positions of the narrow components are marked with upward arrows in the bottom of each panel. The bottom panel shows the covering factors with their $1 \sigma$ error bars, measured for each narrow component by MINFIT (green circles) or for each pixel (black dots). If we had to assume $C_{\mathrm{f}}=1$ for some components because of unphysical $C_{\mathrm{f}}$ values derived from MINFIT (see Section 3.2), we do not plot them.

(The complete figure set (58 images) is available.)

Table 4

Statistical Properties of Poisson Systems of NALs

\begin{tabular}{|c|c|c|c|c|c|c|c|c|c|c|}
\hline & & \multicolumn{3}{|c|}{ C IV } & \multicolumn{3}{|c|}{$\mathrm{N} \mathrm{v}$} & \multicolumn{3}{|c|}{ Si IV } \\
\hline & & $\overline{\mathrm{AAL}^{\mathrm{a}}}$ & Non-AAL ${ }^{\mathrm{b}}$ & Total & $\mathrm{AAL}^{\mathrm{a}}$ & Non-AAL ${ }^{b}$ & Total & $\overline{\mathrm{AAL}^{\mathrm{a}}}$ & Non-AAL ${ }^{b}$ & Total \\
\hline \multirow[t]{2}{*}{ Path length } & $\delta z^{c}$ & 0.05 & 0.56 & 0.61 & 0.05 & 0.01 & 0.07 & 0.05 & 0.37 & 0.42 \\
\hline & $\delta \beta^{\mathrm{c}}$ & 0.02 & 0.19 & 0.21 & 0.02 & 0.00 & 0.02 & 0.02 & 0.12 & 0.14 \\
\hline \multirow[t]{3}{*}{ Image A } & $N^{\mathrm{d}}$ & $3_{-1.6}^{+2.9}$ & $9_{-2.9}^{+4.1}$ & $12_{-3.4}^{+4.6}$ & $2_{-1.3}^{+2.6}$ & $0_{-0}^{+1.8}$ & $2_{-1.3}^{+2.6}$ & $0_{-0}^{+1.8}$ & $3_{-1.6}^{+2.9}$ & $3_{-1.6}^{+2.9}$ \\
\hline & $d N / d z$ & $57_{-31}^{+55}$ & $16_{-5.3}^{+7.3}$ & $20_{-5.6}^{+7.4}$ & $38_{-24}^{+50}$ & $0_{-0}^{+132}$ & $30_{-19}^{+39}$ & $0_{-0}^{+35}$ & $8.1_{-4.4}^{+7.9}$ & $7.1_{-3.9}^{+6.9}$ \\
\hline & $d N / d \beta$ & $177_{-96}^{+172}$ & $47_{-16}^{+22}$ & $58_{-17}^{+22}$ & $118_{-76}^{+155}$ & $0_{-0}^{+409}$ & $93_{-60}^{+123}$ & $0_{-0}^{+108}$ & $25_{-14}^{+24}$ & $22_{-12}^{+21}$ \\
\hline \multirow[t]{3}{*}{ Image B } & $N^{\mathrm{d}}$ & $3_{-1.6}^{+2.9}$ & $8_{-2.8}^{+4.0}$ & $11_{-3.3}^{+4.4}$ & $2_{-1.3}^{+2.6}$ & $0_{-0}^{+1.8}$ & $2_{-1.3}^{+2.6}$ & $0_{-0}^{+1.8}$ & $3_{-1.6}^{+2.9}$ & $3_{-1.6}^{+2.9}$ \\
\hline & $d N / d z$ & $57_{-31}^{+55}$ & $14_{-4.9}^{+7.1}$ & $18_{-5.3}^{+7.2}$ & $38_{-24}^{+50}$ & $0_{-0}^{+132}$ & $30_{-19}^{+39}$ & $0_{-0}^{+35}$ & $8.1_{-4.4}^{+7.9}$ & $7.1_{-3.9}^{+6.9}$ \\
\hline & $d N / d \beta$ & $177_{-96}^{+172}$ & $42_{-15}^{+21}$ & $53_{-16}^{+21}$ & $118_{-76}^{+155}$ & $0_{-0}^{+409}$ & $93_{-60}^{+123}$ & $0_{-0}^{+108}$ & $25_{-14}^{+24}$ & $22_{-12}^{+21}$ \\
\hline \multirow[t]{2}{*}{$\mathrm{M} 07 \mathrm{a}^{\mathrm{e}}$} & $d N / d z$ & 14. & 6.6 & 6.9 & 5.2 & 0.0 & 4.6 & 3.2 & 3.7 & 3.7 \\
\hline & $d N / d \beta$ & 54. & 24. & 25. & 21. & 0.0 & 18. & 13. & 14. & 14. \\
\hline
\end{tabular}

Notes.

${ }^{a}$ Associated absorption lines with $v_{\mathrm{ej}} \leqslant 5000 \mathrm{~km} \mathrm{~s}^{-1}$ from the quasar emission redshift.

${ }^{\mathrm{b}}$ Non-associated absorption lines with $v_{\mathrm{ej}}>5000 \mathrm{~km} \mathrm{~s}^{-1}$ from the quasar emission redshift.

${ }^{\mathrm{c}}$ Total redshift and speed intervals considered in the determination of $d N / d z$ and $d N / d \beta$.

${ }^{\mathrm{d}}$ Number of Poisson systems (i.e., groups of NALs that lie within $200 \mathrm{~km} \mathrm{~s}^{-1}$ of each other).

e $d N / d z$ and $d N / d \beta$ values from Misawa et al. (2007a).

detailed velocity structure of PALs using higher quality spectra with a $\mathrm{S} / \mathrm{N}$ double that of the past observations with Subaru. We first confirm the line-locking pattern in velocity plots of the C IV PAL as shown in Figure 11, which was already noted in Misawa et al. (2013). Like the other NAL absorbers, the PAL absorbers also appear to be outflowing almost parallel to our sightline. We also reconfirm that the CIV PALs became shallower (i.e., decreasing in equivalent width) over a large velocity range of the profile between epochs E1 and E2/E3 (see Figure 11) at $\Delta t_{\text {rest }}$ $\sim 1.3$ years in the quasar rest-frame. However, these lines are almost stable on the shorter timescales of $\Delta t_{\text {rest }} \sim 0.05$ years between epochs E2 and E3. The broad spectral coverage of the VLT/UVES spectra allows us to detect for the first time the $\mathrm{O}$ VI doublet corresponding to the PAL absorber (see Figure 10). There is also a hint of the Si Iv doublet detected, but it appears blended with other lines at lower redshift. 


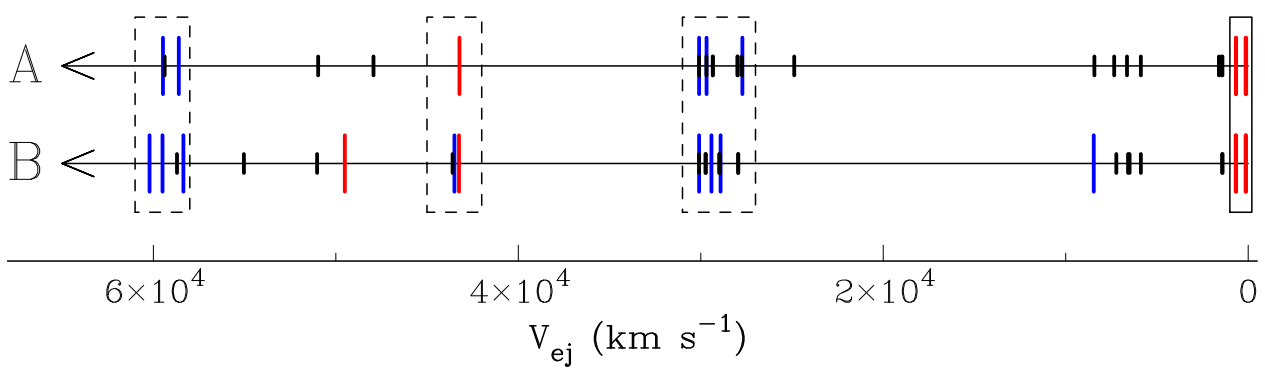

Figure 4. Ejection velocity distribution of classes A (red line), B (blue line), and C (black line) NALs and PALs along the sightlines to the images A and B. All Mg II NALs are located outside of the range of the velocity plot. Each of the red lines at $v_{\mathrm{ej}} \sim 0 \mathrm{~km} \mathrm{~s}^{-1}$ surrounded by a solid rectangle includes both C iv and $\mathrm{N} v \mathrm{PALs}$, respectively. Three clustering regions are surrounded by dotted rectangles.

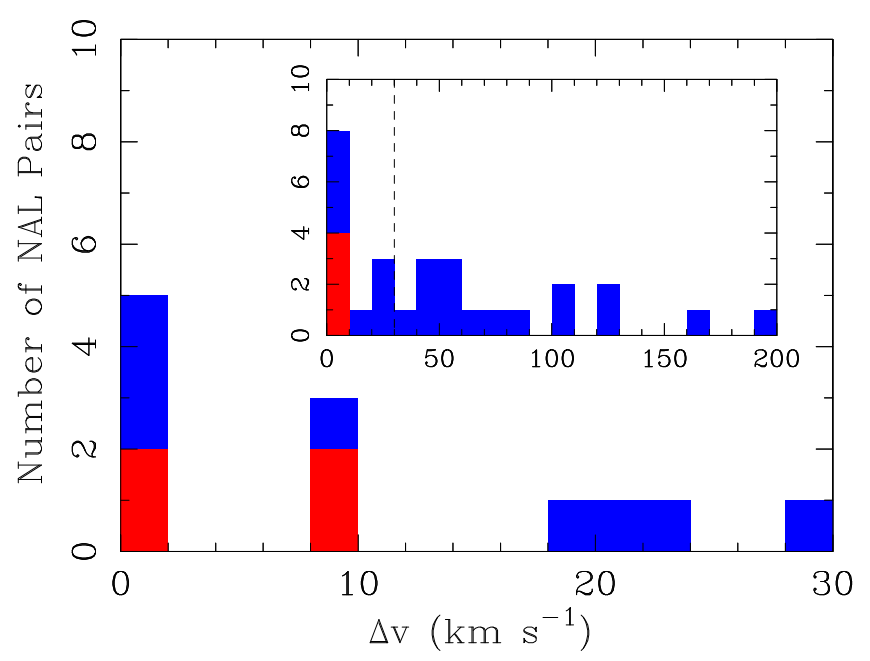

Figure 5. Distribution of velocity offset $(\Delta v)$ between PALs (red histogram) and class A/B NALs (blue histogram) in the two different images (image A and B) that match each other within $\Delta v \leqslant 200 \mathrm{~km} \mathrm{~s}^{-1}$, as discussed in Section 4.1.4.

\subsubsection{Comparison of Covering Factors}

In addition to line profiles and strengths, we also compare covering factors for the clean part of the $\mathrm{C}$ IV and $\mathrm{N} v$ PALs in the three epochs. The fitting parameters to the PALs in epoch E2 with MINFIT are summarized in Table 3 . The total column densities of $\mathrm{C}_{\mathrm{IV}}$ and $\mathrm{N} v$ PALs after summing all Voigt components are $\log \left(N_{\mathrm{N} \mathrm{V}} / \mathrm{cm}^{-2}\right)=15.62$ and $\log \left(N_{\mathrm{C} \text { IV }} / \mathrm{cm}^{-2}\right)=15.90 \quad$ in image A and 16.04 and 15.89 in the image B spectrum. These values are all consistent with the corresponding values that we measured in epoch E1 (Misawa et al. 2013) with differences of a factor of $\leqslant 2$. Because all PALs have small offset velocities and are located on the BELs in the spectra, we should consider the BELRs as well as the continuum source as the background flux source. Because the CIV PAL is partially self-blended (i.e., blending of the blue members of one doublet with the red member of another doublet), we can compare covering factors effectively only for the narrow PALs at $v_{\mathrm{ej}} \sim-100-0 \mathrm{~km} \mathrm{~s}^{-1}$ (corresponding to $\Delta v \sim 400-500 \mathrm{~km} \mathrm{~s}^{-1}$ in Figure 12), for which self-blending is negligible. ${ }^{16}$

\footnotetext{
${ }^{16}$ Negative ejection velocity for these components could be due to our underestimation of these values because the emission redshift is determined from broad UV emission lines, as was done for this quasar by Inada et al. (2006), which are systematically blueshifted from the systemic redshift that is measured by narrow, forbidden lines (see, e.g., Corbin 1990; Tytler \& Fan 1992; Brotherton et al. 1994; Marziani et al. 1996) by about $260 \mathrm{~km} \mathrm{~s}^{-1}$.
}

We show the fitting results for the PALs in epoch E2 in Figure 12 and Table 3 . The average covering factors of $\mathrm{C}$ IV and $\mathrm{N} v$ at $v_{\mathrm{ej}} \sim-100-0 \mathrm{~km} \mathrm{~s}^{-1}$ (i.e., $\Delta v \sim 400-500 \mathrm{~km} \mathrm{~s}^{-1}$ in Figure 12) are $C_{\mathrm{f}}=0.47 \pm 0.03$ and $0.58 \pm 0.05$ toward image $\mathrm{A}$, and $C_{\mathrm{f}}=0.23 \pm 0.02$ and $0.39 \pm 0.08$ toward image B. These values are all consistent with the corresponding values in epoch E1 toward image A $\left(C_{\mathrm{f}}=0.47 \pm 0.05\right.$ and $\left.0.61 \pm 0.07\right)$ and image $\mathrm{B}\left(C_{\mathrm{f}} \sim 0.2 \text { and } 0.35 \pm 0.05\right)^{17}$, and in epoch E3 toward image $\mathrm{A}\left(C_{\mathrm{f}}=0.45 \pm 0.09\right.$ and $\left.0.54 \pm 0.09\right)$ and image B $\left(C_{\mathrm{f}}=0.20 \pm 0.05\right.$ and $\left.0.34 \pm 0.19\right)$. Thus, covering factors are not variable at least on a timescale of $\Delta t_{\text {rest }} \sim 1.3$ years.

The $\mathrm{N} v$ PALs all have larger $C_{\mathrm{f}}$ values than that for $\mathrm{C}_{\mathrm{IV}}$, which is consistent with past results that higher ionization transitions tend to have larger coverage fractions (e.g., Petitjean \& Srianand 1999; Srianand \& Petitjean 2000; Misawa et al. 2007a; Muzahid et al. 2015). This result suggests that i) the size (i.e., distance from the central black hole) of the $\mathrm{N} v$ BELR is smaller than that of the C IV BELR, and/or ii) the size of the $\mathrm{N} \mathrm{v}$ absorbers is larger than that of the $\mathrm{C}$ Iv absorbers. It is also interesting that the PALs in the image A spectrum always have larger $C_{\mathrm{f}}$ values than those in the image B spectrum, which is additional evidence for variations in the structure along multiple sightlines.

\section{DISCUSSION}

\subsection{Velocity Anisotropies in NAL Absorbers}

Line-locking is seen in both NALs and PALs, thus we are likely to be observing the quasar almost along the direction of the outflow. Nonetheless, we discovered a velocity difference between the two sightlines (see Table 2). Given the redshifts of the lens $\left(z_{1}=0.58\right.$; Oguri et al. 2008) and the source $\left(z_{\mathrm{s}}=2.197\right)$, the separation angle of the light rays that form images $\mathrm{A}$ and $\mathrm{B}$, as seen from the source ${ }^{18}$, is $\theta^{\prime} \sim 14$ " 6 . This velocity gradient suggests that the outflowing wind has internal velocity anisotropies on a scale of $\sim 1 \mathrm{~km} \mathrm{~s}^{-1} \operatorname{arcsec}^{-1}$ on average. Indeed, the hydrodynamic simulations of Proga \& Kallman (2004) show this type of internal velocity variations. A recent radiation-MHD simulation by Takeuchi et al. (2013) also reproduced such velocity variations over a typical spatial scale of $\sim 5 \times 10^{-4} \mathrm{pc}$. This is consistent with the size of NAL absorbers, which is also comparable to the size of the

\footnotetext{
17 Because no absorption component was used for C Iv PAL at $v_{\mathrm{ej}} \leqslant 0 \mathrm{~km} \mathrm{~s}^{-1}$ in the epoch E1 spectrum (Misawa et al. 2013), we adopt an average line depth for this region as a covering factor.

${ }^{18}$ The separation angle as seen by the source is given by $\theta^{\prime}=\left(D_{\mathrm{ol}} / D_{\mathrm{sl}}\right) \times \theta$, where $\theta$ is the observed separation angle of the images (22."5) and $D_{\mathrm{ol}}$ and $D_{\mathrm{sl}}=\left(\left(1+z_{s}\right) /\left(1+z_{l}\right)\right) \times D_{\mathrm{ls}}$ are angular diameter distances from the observer to the lens and from the source to the lens, respectively.
} 

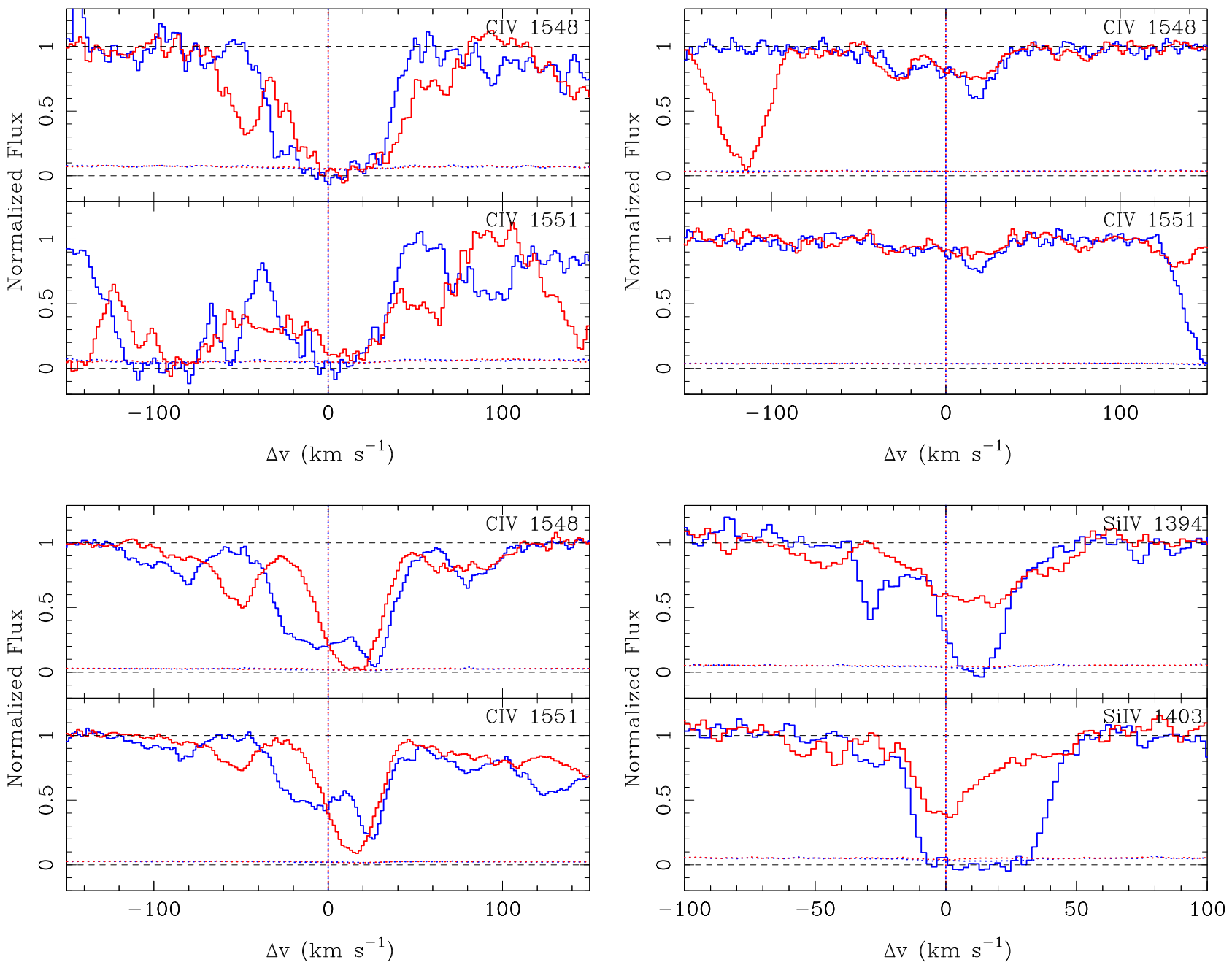

Figure 6. Comparison of spectra and their $1 \sigma$ errors around C Iv NALs at $z_{\mathrm{abs}} \sim 1.8909$ (upper left), 2.1349 (upper right), 2.1819 (lower left), and Si Iv NAL at $z_{\text {abs }} \sim$ 1.8909 (lower right) in image A (blue histogram) and B (red histogram) spectra for systems that have a small relative velocity between the images.

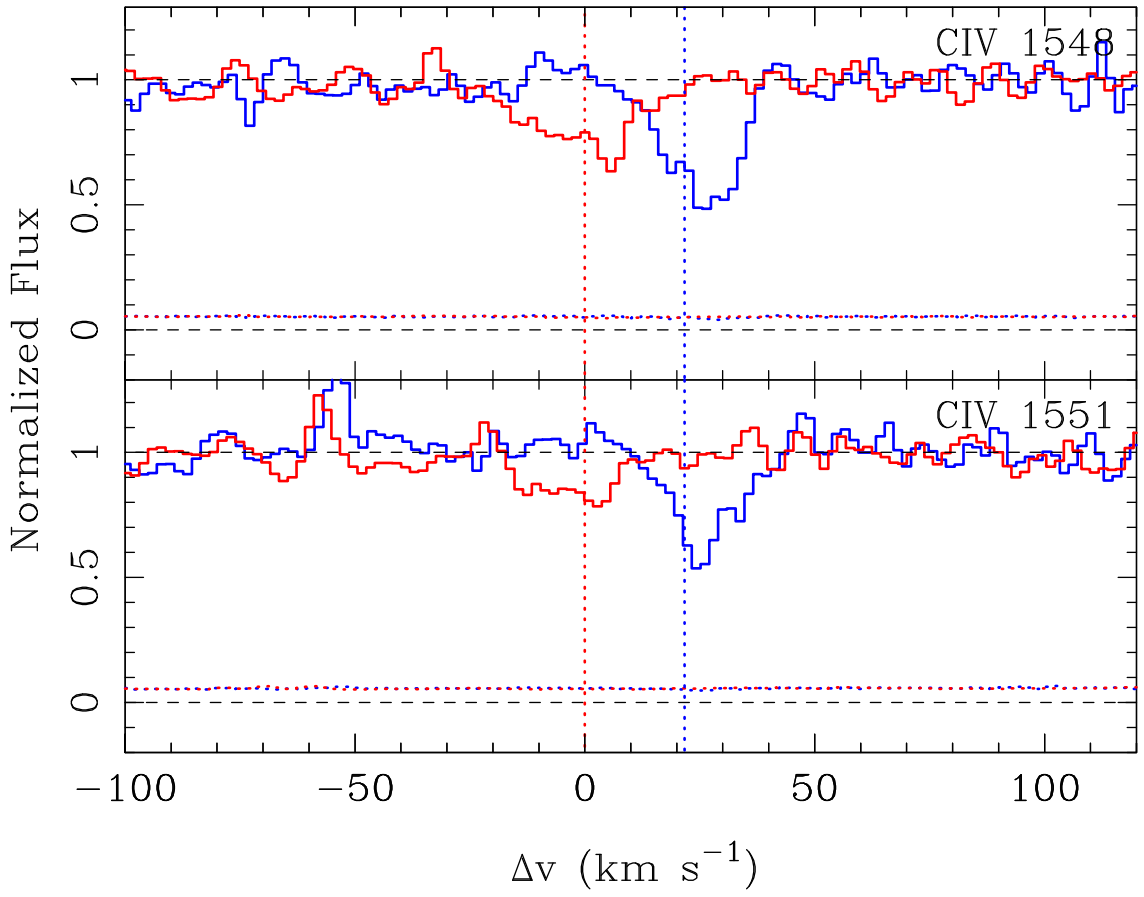

Figure 7. Same as Figure 6 but for class A C iv NALs at $z_{\text {abs }} \sim 1.7652$ in image A (blue histogram) and at $z_{\text {abs }} \sim 1.7650$ in image B (red histogram). The latter corresponds to the system center $\left(\Delta v=0 \mathrm{~km} \mathrm{~s}^{-1}\right)$ in this plot. 


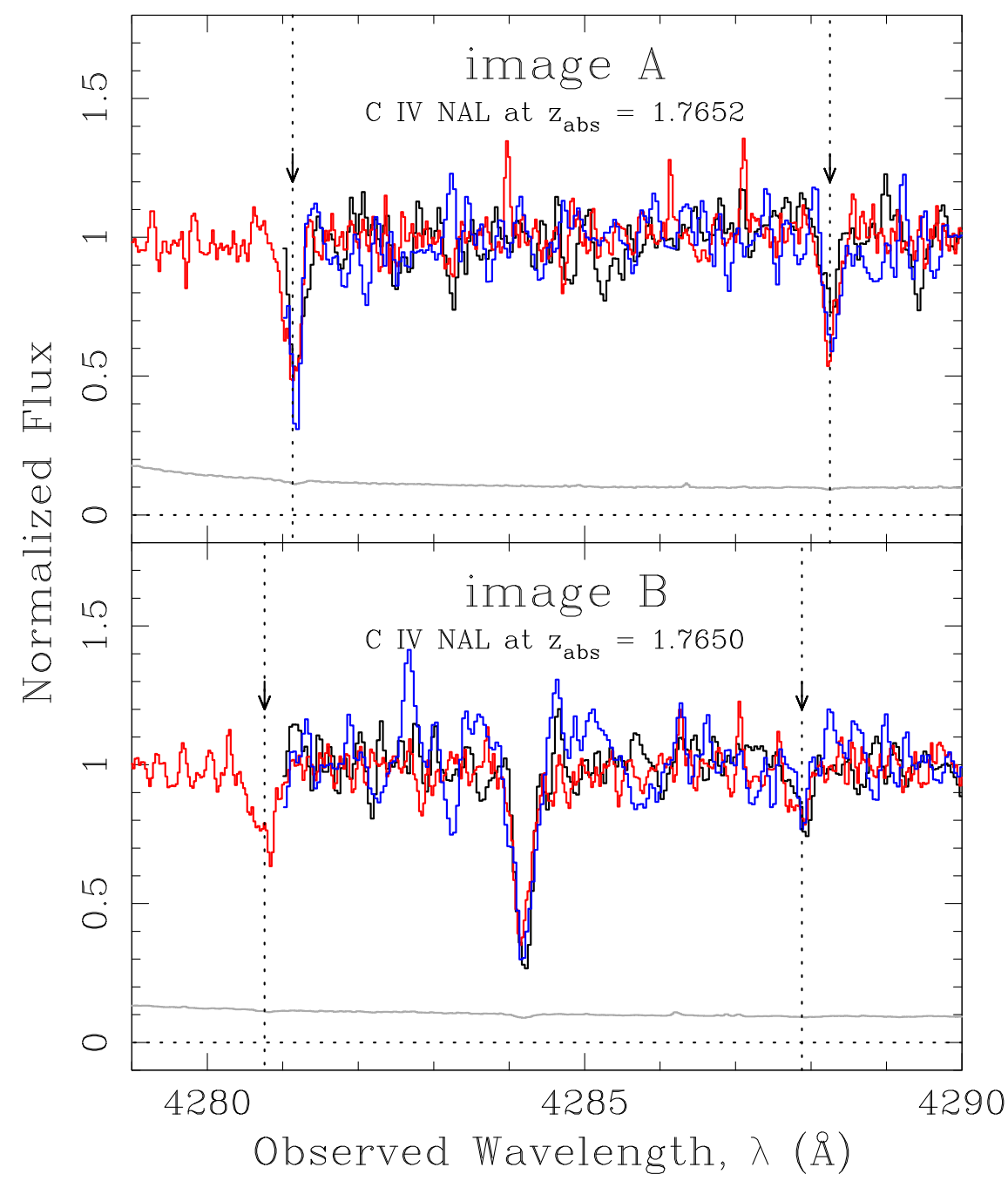

Figure 8. Comparison of $\mathrm{C}$ Iv NALs at $z_{\mathrm{abs}}=1.7652$ in image $\mathrm{A}$ (top panel) and at $z_{\mathrm{abs}}=1.7650$ in image $\mathrm{B}$ (bottom panel). Black, red, and blue histograms denote spectra in epoch E1, E2, and E3, respectively. The positions of blue and red member of the doublet are marked with down arrows and vertical dashed lines. A strong feature at $\lambda \sim 4284 \AA$ in the bottom panel is C IV 1551 at $z_{\mathrm{abs}}=1.7627$. Regions at $\lambda \leqslant 4281 \AA$ are not covered by the Subaru/HDS spectra in epochs E1 and E3 because they are located at the edge of the CCD.

continuum source $\left(\sim 2.5 \times 10^{-4} \mathrm{pc}\right.$; Misawa et al. 2013). However, we should note that the velocity variations in the simulations are found in the inner part of the wind at distances of $\sim 50 R_{\mathrm{g}}$ from the center.

The velocity distribution of intrinsic NALs that are classified into class A or B appears to cluster around values of $v_{\mathrm{ej}}$ $\sim 59,000,43,000$, and $29,000 \mathrm{~km} \mathrm{~s}^{-1}$, except for two intrinsic NALs at $v_{\mathrm{ej}} \sim 49,500 \mathrm{~km} \mathrm{~s}^{-1}$ (class A) and $\sim 8,500 \mathrm{~km} \mathrm{~s}^{-1}$ (class B) in the image B spectrum (Figure 4). These clustering patterns are reminiscent of the filamentary structures obtained by numerical simulations (e.g., Proga et al. 2000). If these patterns are indeed due to filamentary structures, there should exist some velocity anisotropies within them. Velocity dispersions in the three intrinsic NAL clusters are $\delta v \sim 900$, 260 , and $1200 \mathrm{~km} \mathrm{~s}^{-1}$, respectively, which correspond to about $1.6 \%, 0.6 \%$, and $4.0 \%$ of their average ejection velocity.

\subsection{Ionization Condition in PAL Absorbers}

The Ly $\alpha, \mathrm{C}_{\text {IV }}$, and $\mathrm{N}$ v PALs have been monitored at three epochs (E1, E2, and E3) between 2010 February and 2014 April. Between epochs E1 and E2/E3, C Iv PALs show a clear variation in their strength (i.e., depth). There are several possible reasons for this time variability: (1) gas motion across our line of sight (e.g., Gibson et al. 2008; Hamann et al. 2008; Muzahid et al. 2015); (2) changes in the ionization state of the absorber (e.g., Misawa et al. 2007b; Hamann et al. 2011); and (3) redirection of photons around the absorber by scattering material (e.g., Lamy \& Hutsemékers 2004; Misawa et al. 2010). Among these, the first scenario can be rejected because all absorption components in the C IV PAL vary in concert, which requires the implausible situation in which all clouds cross our sightline simultaneously (e.g., Misawa et al. 2007b). The third scenario is also less likely because it requires a variation in the covering factor while the $C_{\mathrm{f}}$ values remain almost stable both in $\mathrm{C}$ Iv and $\mathrm{N} v$ PALs between epochs $\mathrm{E} 1$ and E2/E3. Thus, only the scenario involving a change in ionization state deserves further investigation. This scenario is further separated into two variants: i.e., $\mathrm{C}^{3+}$ ions are ionized to $\mathrm{C}^{4+}$ or they recombine to $\mathrm{C}^{2+}$. Both variants of this scenario can explain the decreasing EW of the $\mathrm{C}_{\mathrm{IV}}$ PALs. Without knowing an absorber's ionization parameter ${ }^{19}$, we cannot tell

\footnotetext{
19 The ionization parameter $U$ is defined as the ratio of hydrogen ionizing photon density $\left(n_{\gamma}\right)$ to the electron density $\left(n_{e}\right)$.
} 

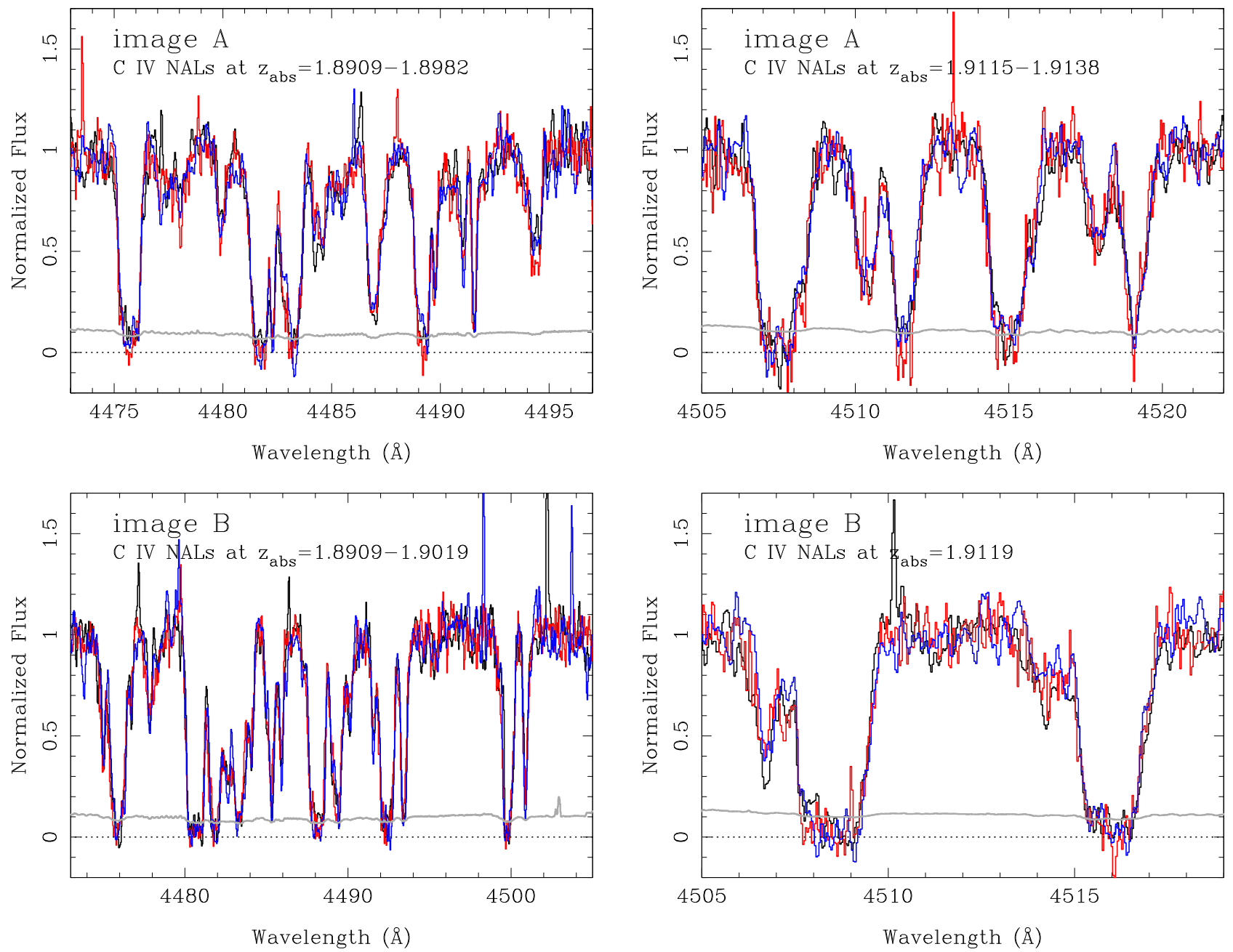

Figure 9. Comparison of spectra and their averaged $1 \sigma$ errors around $\mathrm{C}$ iv NALs at $z_{\mathrm{abs}} \sim 1.8909-1.8982$ in image A (upper left), 1.9115-1.9138 in image A (upper right), 1.8909-1.9019 in image B (lower left), and 1.9119 in image B (lower right) taken in epoch E1 (black histogram), E2 (red histogram), and E3 (blue histogram).

which one is more likely. If the latter variant applies, we can place constraints on the electron density and the distance from the ionizing photon source by the same prescription as used in Narayanan et al. (2004), taking the variability timescale as an upper limit to the recombination time. Based on the observation that the CIV PALs vary between epochs E1 and E3 over $\Delta t_{\text {obs }}=1514$ days (i.e., $\Delta t_{\text {rest }}=474$ days) ${ }^{20}$ we can place a lower limit on the electron density of the absorber as $n_{e}>8.7 \times 10^{3} \mathrm{~cm}^{-3}$, and an upper limit on the distance from the flux source as $r<620 \mathrm{pc}$, assuming $U \sim 0.02$, the value at which the $\mathrm{C}_{\mathrm{IV}}$ and $\mathrm{N} v$ ions are close to the optimal ionization states for those elements (e.g., Hamann et al. 1995).

\subsection{Global Picture of the Outflow from SDSS J1029+2623}

In Table 5, we summarize the physical properties of broad PALs, narrow PALs, and intrinsic NALs. We also present a possible geometry of the outflow along our two sightlines in Figure 13 based on our previous constraints.

First, we see almost the same absorption profiles of Ly $\alpha$, $\mathrm{N} v$, and $\mathrm{C}$ Iv PALs in the two lensed images except for a clear

\footnotetext{
${ }^{20}$ We compare spectra in epochs E1 and E3 instead of epochs E1 and E2 because the E2 observation spans the time range 2014 January 28 to February 26 , which gives an additional uncertainty for measuring the variation timescale.
}

difference in the narrow PALs at $v_{\mathrm{ej}} \sim-100-0 \mathrm{~km} \mathrm{~s}^{-1}$. We also confirm that none of the intrinsic NALs have common absorption profiles between the images. These results suggest that the size of the broad PAL absorbers are larger than the projected separation between sightlines at the distance of the absorbers, $r \theta$, while the narrow PAL absorbers and NAL absorbers have sizes smaller than $r \theta$. Such common absorption profiles are, however, observable regardless of the absorber's size, if their distance from the flux source is smaller than the boundary radius $r_{\mathrm{b}}$ at which the two sightlines of lensed images become fully separated with no overlap (Misawa et al. 2013). The boundary distance is $\sim 3.5 \mathrm{pc}$ for SDSS J1029+2623 if only the continuum source is counted. If we also consider the BELR (whose size is estimated to be $\sim 0.09 \mathrm{pc})^{21}$ as the background source, $r_{\mathrm{b}}$ would be $\sim 1200 \mathrm{pc}$ (see Figure 13).

We can also place constraints on the absorber size based on partial covering analysis. Intrinsic NALs with large ejection velocities have partial coverage, although they absorb only the continuum photons. This means their physical scale is comparable to or smaller than the size of the continuum source, $d_{\text {cloud }} \leqslant 2.5 \times 10^{-4} \mathrm{pc}$. On the other hand, broad PAL

\footnotetext{
$\frac{21}{21}$ This is calculated by Misawa et al. (2013) based on the empirical equation in McLure \& Dunlop (2004).
} 


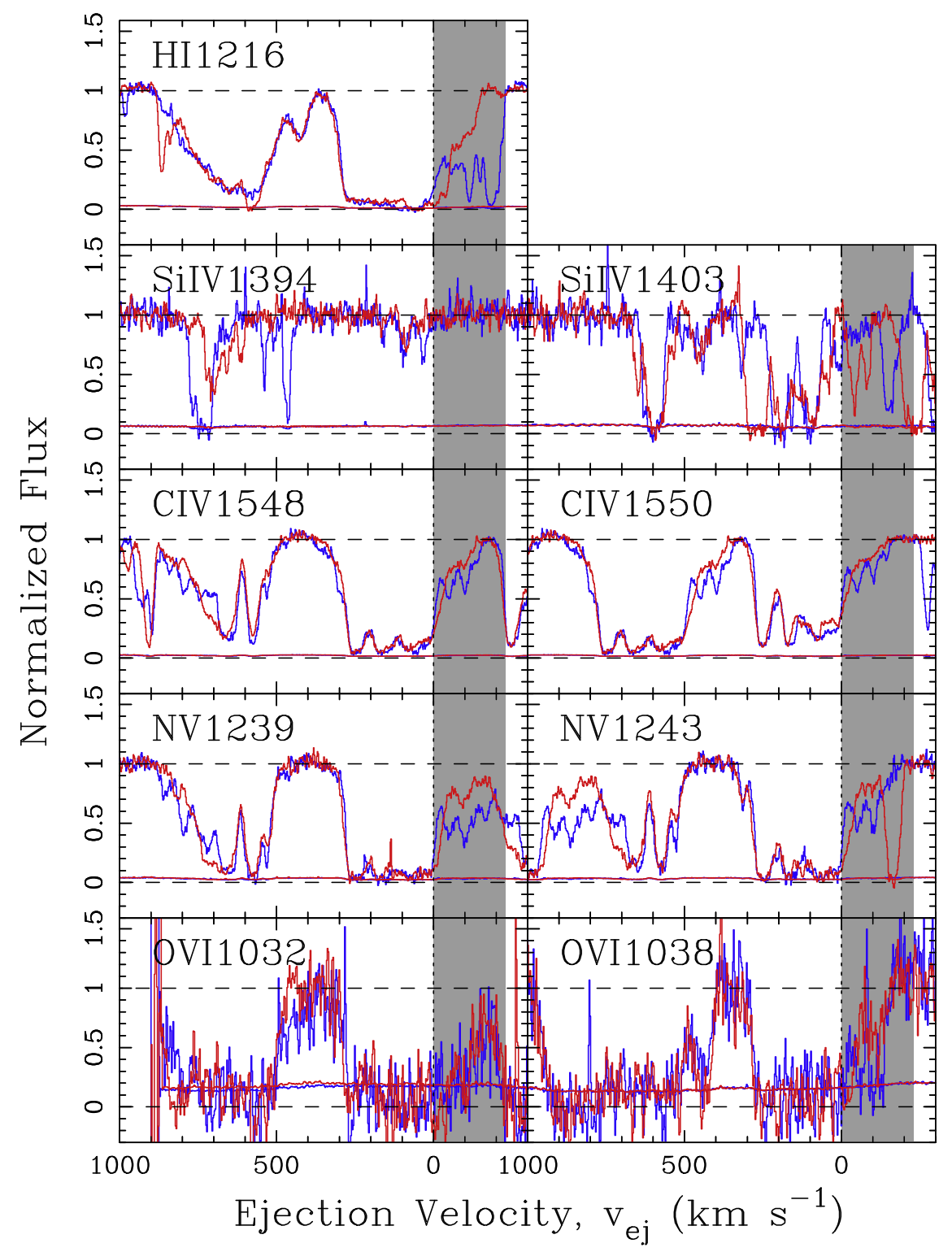

Figure 10. Comparison of normalized spectra around $\mathrm{Ly} \alpha, \mathrm{Si}$ Iv, $\mathrm{C}$ Iv, $\mathrm{N}$ v, and $\mathrm{O}$ vi PALs detected in the images A (blue) and B (red). The horizontal axis is the offset velocity from the quasar emission redshift, and the vertical axis is the normalized flux. The histograms above the zero flux line are $1 \sigma$ flux errors. Due to heavy blending with other lines at lower redshift, the existence of the Si Iv doublet may not be present. The O vI 1032 is located at the edge of the observed spectrum. Absorption features at $v_{\mathrm{ej}}<0 \mathrm{~km} \mathrm{~s}^{-1}$ in the shaded areas are narrow PALs, while the other features at $v_{\mathrm{ej}}>0 \mathrm{~km} \mathrm{~s}^{-1}$ are broad PALs.

absorbers cover almost entirely both the BELR and the continuum source, which means the size of the absorbers as a whole is comparable to or larger than the BELR size, $\geqslant 0.09$ pc. Narrow PAL absorbers may consist of a number of small clumpy clouds because they show partial coverage.

In our VLT/UVES spectra, we detected high-ionization transitions like $\mathrm{O} v \mathrm{v}$ and $\mathrm{Nv}$ in the PAL systems, while the intrinsic NAL systems are in various ionization states, with or without high-ionization transitions, as already noted in the literature (e.g., Misawa et al. 2007a; Ganguly et al. 2013). Some C IV NAL absorbers also show N v transitions, while others do not. Because Ly $\alpha$ and $\mathrm{N} v$ in the PAL system are less variable than $\mathrm{C}$ IV and because $\mathrm{N} v$ has larger covering factor than $\mathrm{C}$ IV, the cross-section of Ly $\alpha$ and $\mathrm{N} v$ should be larger than that of $\mathrm{C}$ IV. PAL absorbers with strong $\mathrm{O}$ vi lines are probably located much closer to the ionizing flux source than NAL absorbers, although this conclusion depends on the density of the absorber.
It is well known that both broad and narrow absorption lines at $z_{\mathrm{abs}} \sim z_{\mathrm{em}}$ tend to vary (Wise et al. 2004; Misawa et al. 2014a), while NALs with large ejection velocities are rarely variable (e.g., Chen et al. 2015). Indeed, the PALs in our spectra are variable, while most of the C IV NALs are probably stable between the three epochs, as we observed for a few strong C IV NALs. Misawa et al. (2014a) suggest that broader absorption lines like the broad PALs can vary mainly due to a change in their ionization state while NALs like narrow PALs and NALs vary primarily due to the gas motion transverse to our sightlines. The gas motion scenario could explain why narrow PALs are variable but highvelocity NALs are not. Based on the dynamical model of Murray et al. (1995) and more recent investigations (e.g., Misawa et al. 2005; Hall et al. 2011), the absorbers at larger distances from the center have small transverse (i.e., orbital) velocities compared to their radial velocities. If intrinsic NALs lie at larger distances than narrow PALs, their small transverse velocities would rarely lead to 

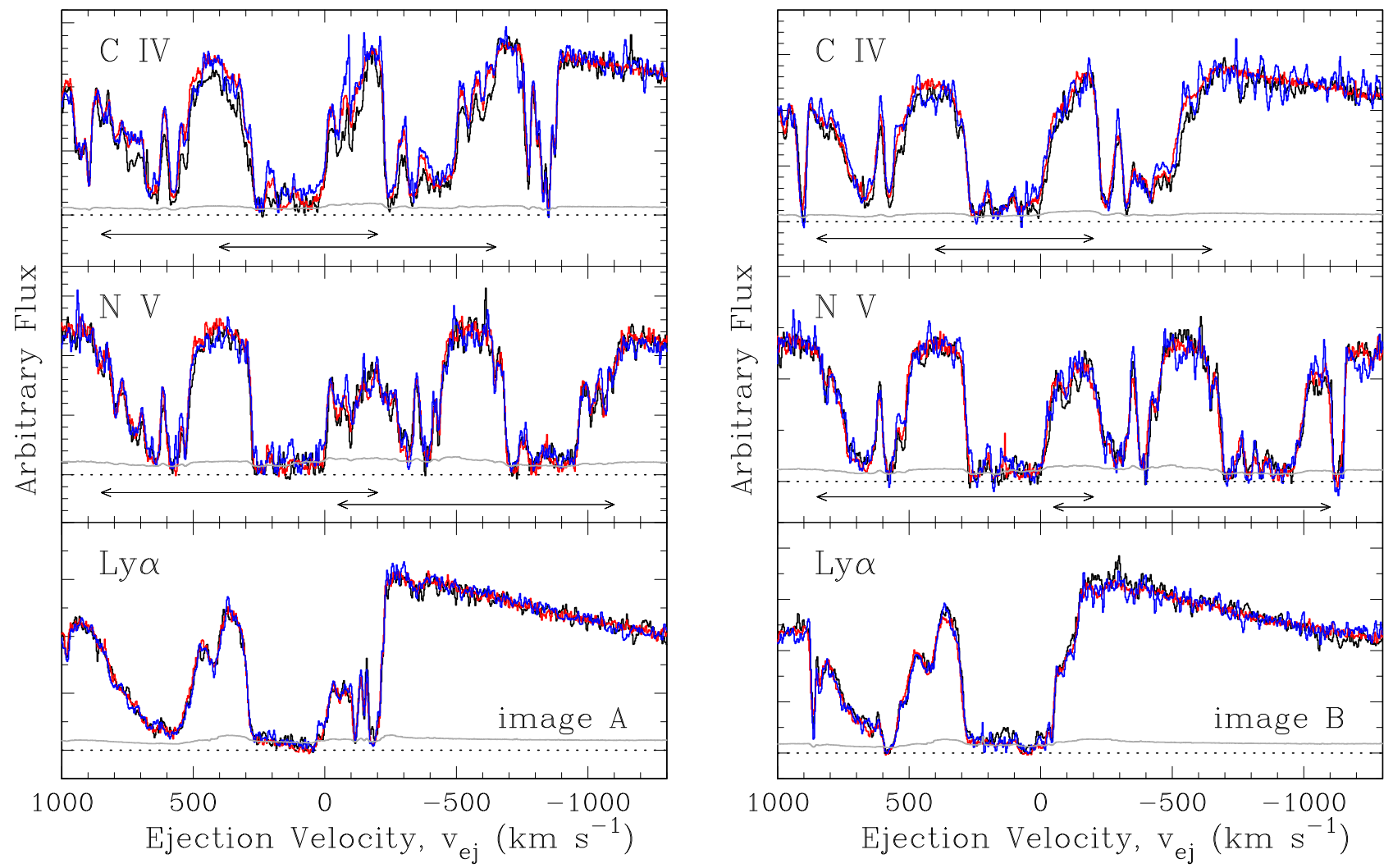

Figure 11. Comparison of $\mathrm{C}$ Iv, $\mathrm{N} \mathrm{v}$, and Ly $\alpha$ PALs at $z_{\mathrm{abs}} \sim z_{\mathrm{em}}$ in images A and B, taken in epochs E1 (black), E2 (red), and E3 (blue). These spectra are scaled to each other by applying a least-squares method for unabsorbed wavelength regions at both sides of the PALs. The vertical axis denotes an arbitrary flux because we did not perform absolute flux calibration without flux standard stars. The gray histograms above the zero flux line are $1 \sigma$ flux errors after taking averages of those in three observing epochs. Double-headed arrows in the bottom of the first two panels mark the velocity ranges of the blue and red members of the C IV and $\mathrm{N} v$ doublets.

time variability, while the large transverse velocities of narrow PALs can lead to time variability more frequently.

\section{SUMMARY}

In this study, we performed a spectroscopic observation for images A and B of the gravitationally lensed quasar SDSS $\mathrm{J} 1029+2623$, and monitored the absorption profiles in these spectra as well as in the previous two observations. Using highquality spectra taken with VLT/UVES, we detected intrinsic NALs as well as broader, PALs, and fit models to the line profiles. Based on the results of our multi-sightline spectroscopy, we discuss a possible geometry and internal structure of the outflowing wind along our sightlines. Our main results are as follows.

1. We detected 66 NALs, of which 24 are classified as intrinsic NALs (physically associated with the quasar) based on partial coverage analysis.

2. Class A and B NALs cluster at $v_{\mathrm{ej}} \sim 59,000,43,000$, and $29,000 \mathrm{~km} \mathrm{~s}^{-1}$, which is reminiscent of the filamentary structures that are often obtained in numerical simulations.

3. Our multiple sightline observation suggests that the size of the broad PAL absorbers are larger than the projected distance between sightlines $(r \theta)$ while the narrow PAL absorbers and intrinsic NAL absorbers have sizes smaller than $r \theta$ if their radial distances are greater than the boundary distance.
4. While PAL systems show only high-ionization transitions, including $\mathrm{O}$ VI, intrinsic NAL systems show a wide range of ionization conditions with and without lowionization transitions like $\mathrm{O}_{\mathrm{I}}, \mathrm{Al} \mathrm{II}$, and $\mathrm{Si}$ II, as noted in the literature.

5. No class A/B NALs (i.e., candidates for intrinsic NALs) have common absorption profiles in the two lensed images, which means that the outflow has an internal velocity structure whose typical spatial scale is smaller than the physical distance between the sightlines (i.e., $\leqslant r \theta$ ).

6. Short-time variation in the PALs is probably due to a change in the ionization state of the gas. If this is the case, we can place a lower limit on the gas density as $n_{e} \geqslant$ $8.7 \times 10^{3} \mathrm{~cm}^{-3}$ and an upper limit on the absorber's distance from the flux source as $r \leqslant 620 \mathrm{pc}$.

7. Based on our best knowledge, we present a possible geometry of the outflow along our two sightlines, in which we identify different structures in the outflowing wind that can produce, respectively, broad PALs, narrow PALs, and NALs with large ejection velocities.

For further investigations of the outflow's internal structure, especially in the transverse direction, we should perform the same observations for image C of SDSS J1029+2623. We also aim to observe several lensed quasars with smaller separation angles $\left(\theta \sim 2^{\prime \prime}\right)$ by a single massive galaxy to examine $\sim 10$ times finer internal structure in an outflow, as already mentioned in Misawa et al. (2014b). 

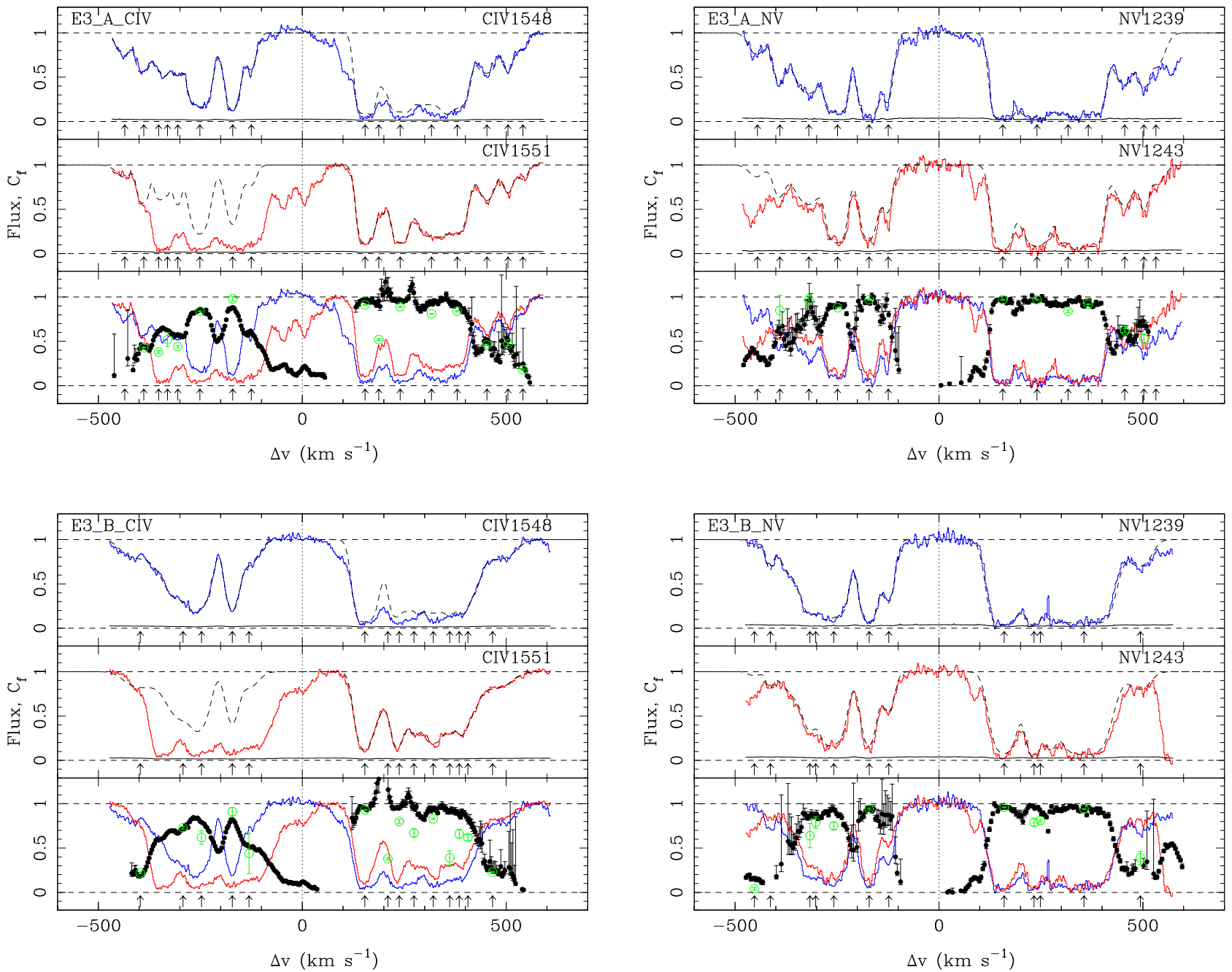

Figure 12. Same figures as Figure 3, but for $\mathrm{C}$ iv and $\mathrm{N}$ v PALs at $z_{\mathrm{abs}} \sim z_{\mathrm{em}}$. We adopt $z=2.1927$ as a system center only for the purpose of displaying these velocity plots. Because C Iv PALs are self-blended, we fit the profiles of the blue and red members of the doublet simultaneously by multiplying the contributions from them (Misawa et al. 2007b). Spectral regions in the top two panels, for which the profiles of the blue and red members of a doublet (blue and red histograms) and the model profile (dashed line) do not match, suffer from self-blending.

Table 5

Properties of PALs and Intrinsic NALs

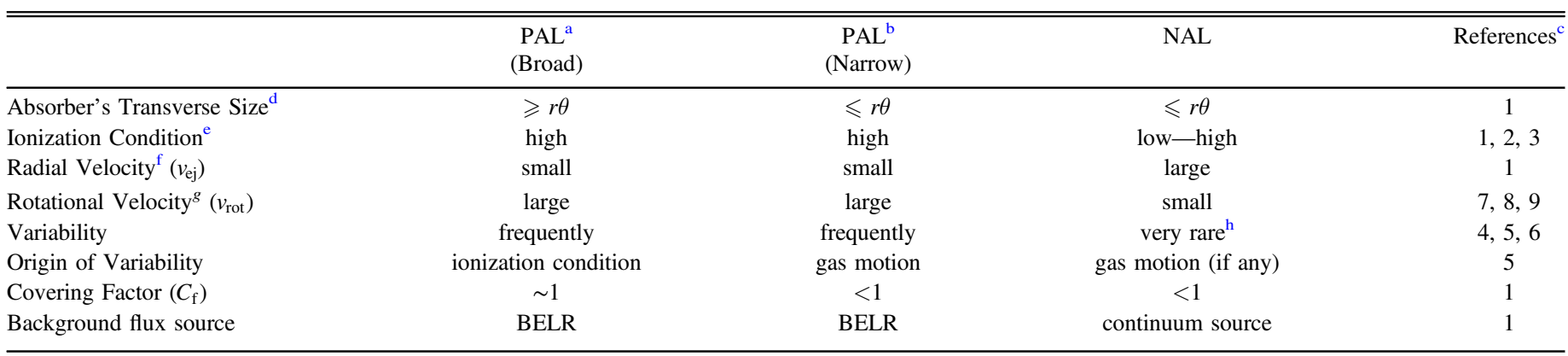

${ }^{a}$ PALs at $v_{\mathrm{ej}}>0 \mathrm{~km} \mathrm{~s}^{-1}$ with common absorption profiles in both sightlines.

${ }^{\mathrm{b}}$ PALs at $v_{\mathrm{ej}}<0 \mathrm{~km} \mathrm{~s}^{-1}$ with sightline variation.

${ }^{c}$ References. (1) This paper, (2) Misawa et al. (2007a), (3) Ganguly et al. (2013) (4) Wise et al. (2004), (5) Misawa et al. (2014a), (6) Chen et al. (2015), (7) Murray et al. (1995), (8) Misawa et al. (2005), (9) Hall et al. (2011).

$\mathrm{d}$ This depends on whether sightline difference is observed (i.e., $\leqslant r \theta$ ) or not (i.e., $\geqslant r \theta$ ).

${ }^{\mathrm{e}}$ Low-, intermediate, and high-ionization transitions are defined in Section 4.1.3.

${ }^{\mathrm{f}}$ Ejection velocity assuming $z_{\mathrm{em}}=2.197$.

g Absorbers with small orbital radius tend to have larger rotational velocity (see Section 5.3).

${ }^{\mathrm{h}}$ Variation probability is very low for high ejection velocity NALs (Chen et al. 2015), although we confirmed this trend only for a few strong C Iv NALs. 


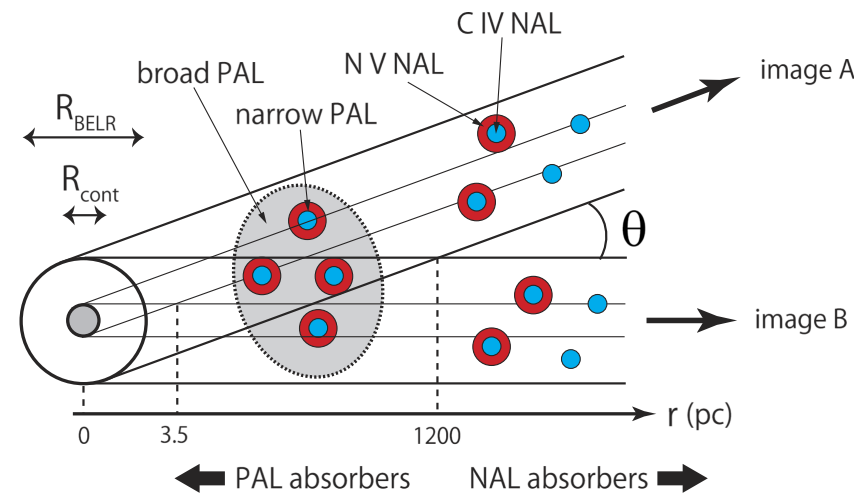

Figure 13. Possible geometry of the outflowing wind along our two sightlines toward the lensed quasar SDSS J1029+2623 based on our observations in three epochs. The gray filled circle corresponds to the broad PAL absorber with the minimum acceptable size, while blue and red filled circles are $\mathrm{C}$ IV and $\mathrm{N} v$ absorbing regions in clumpy clouds as candidates for narrow PAL absorber and intrinsic NALs. The boundary radius of the continuum source $(3.5 \mathrm{pc})$ and BELR (1200 pc) are marked with dashed lines. The NAL absorber's distance is not necessarily larger than $1200 \mathrm{pc}$, although it is at least larger than the distance of the PAL absorbers. We adopt 14 ". 6 as the separation angle between two sightlines seen from the source, while Misawa et al. $(2013,2014 \mathrm{~b})$ used 22 " 5 assuming it is very similar to that seen from us. See Section 5.3 for details.

We thank the anonymous referee for comments that helped us improve the paper. We thank Masamune Oguri, Poshak Gandhi, and Chris Culliton for their valuable comments. We also thank Christopher Churchill for providing us with the MINFIT and SEARCH software packages. The research was supported by the Japan Society for the Promotion of Science through Grant-in-Aid for Scientific Research 15K05020, JGCS Scholarship Foundation, and partially supported by MEXT Grant-in-Aid for Scientific Research on Innovative Areas (No. 15H05894). C.S. acknowledges support from CONICYT-Chile through Becas Chile 74140006. F.E.B. acknowledges support from CONICYT-Chile (Basal-CATA PFB-06/2007, FONDECYT Regular 1141218, "EMBIGGEN" Anillo ACT1101) and the Ministry of Economy, Development, and Tourism's Millennium Science Initiative through grant IC120009, awarded to The Millennium Institute of Astrophysics, MAS. J.C.C. and M.E. acknowledge support from the National Science Foundation through award AST-1312686.

\section{REFERENCES}

Barlow, T. A., \& Sargent, W. L. W. 1997, AJ, 113, 136

Benn, C. R., Carballo, R., Holt, J., et al. 2005, MNRAS, 360, 1455

Bergeron, J., Petitjean, P., Sargent, W. L. W., et al. 1994, ApJ, 436, 33

Blandford, R. D., \& Parne, D. G. 1982, MNRAS, 199, 883

Bowler, R. A. A., Hewett, P. C., Allen, J. T., \& Ferland, G. J. 2014, MNRAS, 445, 359

Brotherton, M. S., Wills, B. J., Steidel, C. C., \& Sargent, W. L. W. 1994, ApJ, 423, 131

Chartas, G., Eracleous, M., Dai, X., Agol, E., \& Gallagher, S. 2007, ApJ, 661,678

Chelouche, D. 2003, ApJL, 596, L43

Chen, Z.-F., Gu, Q.-S., Chen, Y.-M., \& Cao, Y. 2015, MNRAS, 450, 3904

Churchill, C. W. 1997, PhD thesis, Univ. California

Churchill, C. W., Vogt, S. S., \& Charlton, J. C. 2003, AJ, 125, 98

Corbin, M. R. 1990, ApJ, 357, 346

Crenshaw, D. M., Kraemer, S. B., Boggess, A., et al. 1999, ApJ, 516, 750
Ellison, S. L., Ibata, R., Pettini, M., et al. 2004, A\&A, 414, 79

Elvis, M. 2000, ApJ, 545, 63

Emmering, R. T., Bladford, R. D., \& Shlosman, I. 1992, ApJ, 385, 460 Everett, J. E. 2005, ApJ, 631, 689

Fohlmeister, J., Kochanek, C. S., Falco, E. E., et al. 2013, ApJ, 764, 186

Gabel, J. R., Arav, N., \& Kim, T.-S. 2006, ApJ, 646, 742

Ganguly, R., Bond, N. A., Charlton, J. C., et al. 2001, ApJ, 549, 133

Ganguly, R., Eracleous, M., Charlton, J. C., \& Churchill, C. W. 1999, AJ, 117,2594

Ganguly, R., Lynch, R. S., Charlton, J. C., et al. 2013, MNRAS, 435, 1233

Gehrels, N. 1986, ApJ, 303, 336

Gibson, R. R., Brandt, W. N., Schneider, D. P., \& Gallagher, S. C. 2008, ApJ, 675,985

Green, P. J. 2006, ApJ, 644, 733

Hall, P. B., Anosov, K., White, R. L., et al. 2011, MNRAS, 411, 2653

Hamann, F., Barlow, T. A., Beaver, E. A., et al. 1995, ApJ, 443, 606

Hamann, F., Barlow, T. A., \& Junkkarinen, V. 1997a, ApJ, 478, 87

Hamann, F., Barlow, T. A., Junkkarinen, V., \& Burbidge, E. M. 1997b, ApJ, 478,80

Hamann, F., Chartas, G., McGraw, S., et al. 2013, MNRAS, 435, 133

Hamann, F., Kanekar, N., Prochaska, J. X., et al. 2011, MNRAS, 410, 1957

Hamann, F., Kaplan, K. F., Rodríguez Hidalgo, P., Prochaska, J. X., \& Herbert-Fort, S. 2008, MNRAS, 391, L39

Inada, N., Oguri, M., Morokuma, T., et al. 2006, ApJL, 653, L97

Joshi, R., Chand, H., Srianand, R., \& Majumdar, J. 2014, MNRAS, 442,862

Konigl, A., \& Kartje, J. F. 1994, ApJ, 434, 446

Lamy, H., \& Hutsemékers, D. 2004, A\&A, 427, 107

Marziani, P., Sulentic, J. W., Dultzin-Hacyan, D., Calvani, M., \& Moles, M. 1996, ApJS, 104, 37

McLure, R. J., \& Dunlop, J. S. 2004, MNRAS, 352, 1390

Misawa, T., Charlton, J. C., Eracleous, M., et al. 2007a, ApJS, 171, 1

Misawa, T., Charlton, J. C., \& Eracleous, M. 2014a, ApJ, 792, 77

Misawa, T., Eracleous, M., Charlton, J. C., \& Kashikawa, N. 2007b, ApJ, 660,152

Misawa, T., Eracleous, M., Charlton, J. C., \& Tajitsu, A. 2005, ApJ, 629, 115

Misawa, T., Inada, N., Oguri, M., et al. 2014b, ApJL, 794, L20

Misawa, T., Inada, N., Ohsuga, K., et al. 2013, AJ, 145, 48

Misawa, T., Kawabata, K. S., Eracleous, M., Charlton, J. C., \& Kashikawa, N. 2010, ApJ, 719, 1890

Murray, N., Chiang, J., Grossman, S. A., \& Voit, G. M. 1995, ApJ, 451, 498

Muzahid, S., Srianand, R., Arav, N., Savage, B. D., \& Narayanan, A. 2013, MNRAS, 431, 2885

Muzahid, S., Srianand, R., Charlton, J., \& Eracleous, M. 2016, MNRAS, 457, 2665

Narayanan, D., Hamann, F., Barlow, T., et al. 2004, ApJ, 601, 715

Nestor, D., Hamann, F., \& Rodriguez Hidalgo, P. 2008, MNRAS, 386, 2055

Oguri, M., Ofek, E. O., Inada, N., et al. 2008, ApJL, 676, L1

Oguri, M., Schrabback, T., Jullo, E., et al. 2013, MNRAS, 429, 482

Ota, N., Oguri, M., Dai, X., et al. 2012, ApJ, 758, 26

Petitjean, P., \& Srianand, R. 1999, A\&A, 345, 73

Proga, D., \& Kallman, T. R. 2004, ApJ, 616, 688

Proga, D., Stone, J. M., \& Kallman, T. R. 2000, ApJ, 543, 686

Sargent, W. L. W., Boksenberg, A., \& Steidel, C. C. 1988, ApJS, 68, 539

Springel, V., Di Matteo, T., \& Hernquist, L. 2005, ApJL, 620, L79

Srianand, R., \& Petitjean, P. 2000, A\&A, 357, 414

Takeuchi, S., Ohsuga, K., \& Mineshige, S. 2013, PASJ, 65, 88

Tytler, D., Boksenberg, A., Sargent, W. L. W., Young, P., \& Kunth, D. 1987, ApJS, 64, 667

Tytler, D., \& Fan, X.-M. 1992, ApJS, 79, 1

Vestergaard, M. 2003, ApJ, 599, 116

Wampler, E. J., Chugai, N. N., \& Petitjean, P. 1995, ApJ, 443, 586

Weymann, R. J., Morris, S. L., Foltz, C. B., \& Hewett, P. C. 1991, ApJ, 373,23

Weymann, R. J., Williams, R. E., Peterson, B. M., \& Turnshek, D. A. 1979, ApJ, 234, 33

Wise, J. H., Eracleous, M., Charlton, J. C., \& Ganguly, R. 2004, ApJ, 613,129

Young, P. J., Sargent, W. L. W., Boksenberg, A., Carswell, R. F., \& Whelan, J. A. J. 1979, ApJ, 229, 891 